\title{
A POSITIVE PRESERVING HIGH ORDER VFROE SCHEME FOR SHALLOW WATER EQUATIONS: A CLASS OF RELAXATION SCHEMES
}

\author{
CHRISTOPHE BERTHON* AND FABIEN MARCHE ${ }^{\dagger}$
}

\begin{abstract}
The VFRoe scheme has been recently introduced to approximate the solutions of the shallow water equations. One of the main interest of this method is to be easily implemented. As a consequence, such a scheme appears as an interesting alternative to other more sophisticated schemes. The VFRoe methods perform approximate solutions in a good agreement with the expected ones. However, the robustness of this numerical procedure has not been proposed. Following the ideas introduced by Jin-Xin [Comm. Pure Appl. Math., 45, 235-276 (1995)], a relevant relaxation method is derived. The interest of this relaxation scheme is twofold. In the first hand, the relaxation scheme is shown to coincide with the considered VFRoe scheme. In the second hand, the robustness of the relaxation scheme is established and thus the non-negativity of the water height, obtained involving the VFRoe approach, is ensured. Following the same idea, a family of relaxation schemes is exhibited. Next, robust high order MUSCL extensions are proposed. The final scheme is obtained when considering the hydrostatic reconstruction to approximate the topography source terms. Numerical experiments are performed to attest the interest of the procedure.
\end{abstract}

Key words. Positivity preserving, Shallow water equations, VFRoe schemes, Relaxation schemes, High order MUSCL extensions.

AMS subject classifications. 75M12, 35L65, 65M12

1. Introduction. The present work is devoted to the numerical approximations of weak solutions of the shallow water equations, also known as the Saint-Venant equations. When specified in one space dimension, the system under consideration reads as follows:

$$
\left\{\begin{array}{l}
\partial_{t} h+\partial_{x}(h u)=0 \\
\partial_{t}(h u)+\partial_{x}\left(h u^{2}+g h^{2} / 2\right)=-g h d^{\prime}(x)
\end{array}\right.
$$

where $h$ is the local water depth, $u$ is the depth-averaged velocity and $d: \mathbb{R} \rightarrow \mathbb{R}^{+}$ denotes the topography. For the sake of simplicity in the notations, it is convenient to rewrite the system (1.1) in the following condensed form:

$$
\partial_{t} \mathbf{w}+\partial_{x} \mathbf{f}(\mathbf{w})=\mathbf{S}(\mathbf{w}, d)
$$

with

$$
\mathbf{w}=\left(\begin{array}{c}
h \\
h u
\end{array}\right), \quad \mathbf{f}(\mathbf{w})=\left(\begin{array}{c}
h u \\
h u^{2}+\frac{g}{2} h^{2}
\end{array}\right) \quad \text { and } \quad \mathbf{S}(\mathbf{w})=\left(\begin{array}{c}
0 \\
-g h d^{\prime}(x)
\end{array}\right),
$$

where $\mathbf{w}: \mathbb{R} \times \mathbb{R}^{+} \rightarrow \Omega$ is the state vector in conservative variables and $\mathbf{f}(\mathbf{w}): \Omega \rightarrow \mathbb{R}^{2}$ stands for the flux function. The convex set $\Omega$ of the admissible states is defined by:

$$
\Omega=\left\{\mathbf{w} \in \mathbb{R}^{2} ; h \geq 0, u \in \mathbb{R}\right\} .
$$

The homogeneous Saint-Venant system associated with (1.1), given by

$$
\partial_{t} \mathbf{w}+\partial_{t} \mathbf{f}(\mathbf{w})=0,
$$

*Université de Nantes, Laboratoire de Mathématiques Jean Leray, 2 rue de la Houssiniere, BP 92208, 44322 Nantes Cedex 3, France.

${ }^{\dagger}$ I3M, Université Montpellier 2, Place Eugène Bataillon, CC 051, 34000 Montpellier, France. 
is known to be hyperbolic over $\Omega$. As a consequence, the solutions may develop shock discontinuities. In order to rule out the unphysical solutions, the system (1.5) must be supplemented by entropy inequalities [27, 28] (see also [7] and the references therein):

$$
\partial_{t} \tilde{\eta}(\mathbf{w}, d)+\partial_{x} \tilde{G}(\mathbf{w}, d) \leq 0
$$

where

$$
\begin{aligned}
& \eta(\mathbf{w})=h \frac{u^{2}}{2}+\frac{g}{2} h^{2}, \quad G(\mathbf{w})=\left(h \frac{u^{2}}{2}+g h^{2}\right) u \\
& \tilde{\eta}(\mathbf{w}, d)=\eta(\mathbf{w})+h g d, \quad \tilde{G}(\mathbf{w}, d)=G(\mathbf{w})+h g d u .
\end{aligned}
$$

Let us emphasize that the steady state solutions of (1.1) are of primary importance. These specific solutions are given by (for instance, see $[18,13]$ ):

$$
\left\{\begin{array}{l}
h u=\text { cste } \\
\frac{u^{2}}{2}+g h+g d=\text { cste. }
\end{array}\right.
$$

One of them, the steady state of a lake at rest, defined by

$$
u=0, \quad h+d=\text { cste }
$$

plays a crucial role. From a numerical point of view, the considered schemes must satisfy such a property. After the pioneer work of Greenberg et al. [18, 19], schemes that preserve the lake at rest are called well-balanced.

During the last ten years, several schemes have been proposed according to the well-balanced strategy (see [7, 13, 23, 24, 25]). More recently, in [1], a well-balanced hydrostatic reconstruction has been derived. This technique can be applied to any conservative finite volume scheme approximating the homogeneous Saint-Venant system (1.5), to obtain a suitable approximation of the topography source terms. In addition, as soon as the homogeneous system's scheme is robust, the hydrostatic reconstruction preserves the robustness: it preserves the non-negativity of $h$ and it performs relevant approximations of the dry states with $h=0$ (for instance, see also $[33,34]$ for relavant modelisations and approximations of dry areas).

Involving the framework of the hydrostatic reconstruction, we have to consider robust schemes to approximate the weak solutions of (1.5). This avoids some useful but sophisticated strategy (see [13] for a Godunov approach or [7] for a relaxation scheme). Now, when approximating (1.5), several schemes come with the required robustness, the Suliciu relaxation scheme [2, 3, 7] (see also [12, 26] for pioneer works), for instance. However, another type of scheme is frequently used when considering the shallow water flows; namely the VFRoe scheme [14, 15, 17]. Currently, this scheme is known to be very easy to implement, it performs accurate numerical approximations and it is able to deal with dry areas. Unfortunately, no robustness result is established for the VFRoe scheme. This is the aim of the present work. The reader is also refered to the work of Jin-Wen [24, 25], where another type of well-balanced scheme is proposed; namely the interface-type numerical method, which also produces an easy and accurate numerical scheme for the shallow-water equations.

The present paper is organized as follows. In the next section, we recall the derivation of the VFRoe scheme. The derivation is performed when involving the shallow water equations. Section 3 is devoted to relaxation schemes. A relevant relaxation 
model is proposed and analyzed. Following the ideas introduced by Jin-Xin [26] (see also $[2,3,7,12]$ ), the resulting relaxation scheme is shown to coincide with the VFRoe scheme. In other words, the VFRoe scheme is written as a relaxation scheme (see LeVeque-Pelanti [31] where the Roe scheme is seen as a relaxation type method). As a consequence, an approximate Riemann solver, associated with the VFRoe scheme, is known and yields to establish the expected non-negativity of the numerical water depth. Following the same idea, a class of robust relaxation schemes is exhibited. The next section is devoted to the high order extensions. Following an idea introduced by Perthame-Shu [37] (see also [4, 5, 6] for recent developments), we propose high order MUSCL reconstruction which preserves the robustness property. At this level, the robust full discretization of the shallow water equations (1.1) with topography is obtained when considering the hydrostatic reconstruction. In the last section, numerical tests are performed and attest the interest of the proposed numerical procedure.

2. The VFRoe scheme. The VFRoe schemes are approximate Godunov type schemes $[14,15]$. They turn out to be useful approximating the solutions of the shallow water equations since such schemes are able to deal with the dry areas. Moreover, their implementations are very easy. We briefly recall the basis of the VFRoe scheme, proposed in [14], involving non-conservative variables. For the sake of simplicity, we restrict ourselves to regular meshes of size $\Delta x$ such that $\Delta x=x_{i+\frac{1}{2}}-x_{i-\frac{1}{2}}, i \in \mathbb{Z}$, and we note the time step by $\Delta t$ with $t^{n+1}=t^{n}+\Delta t, n \in \mathbb{N}$.

In a more general setting, we turn considering the numerical approximation of hyperbolic system of conservation laws in the form (1.5). To access such an issue, the VFRoe scheme is adopted. This approximate Godunov type scheme is based on the evaluation of the value at the interface between two neighbouring cells when considering the following linearization. First, we adopt an admissible change of variable, $\mathbf{u}=\mathcal{U}(\mathbf{w})$. With some abuse in the notations, we set $\mathcal{W}(\mathbf{u})=\mathbf{w}$ the inverse function of $\mathcal{U}$. Considering smooth enough solutions, the system (1.5) writes as follows:

$$
\partial_{t} \mathbf{u}+B(\mathbf{u}) \partial_{x} \mathbf{u}=0
$$

where $B(\mathbf{u})=\left(\nabla_{\mathbf{u}} \mathcal{W}(\mathbf{u})\right)^{-1} A(\mathcal{W}(\mathbf{u})) \nabla_{\mathbf{u}} \mathcal{W}(\mathbf{u})$ and $A(\mathbf{w})$ is the Jacobian matrix associated with the flux function $\mathbf{f}$. Next, the following linearized Riemann problem is considered:

$$
\begin{aligned}
& \partial_{t} \mathbf{u}+B(\tilde{\mathbf{u}}) \partial_{x} \mathbf{u}=0 \\
& \mathbf{u}(x, 0)=\left\{\begin{array}{lll}
\mathbf{u}_{L}=\mathcal{U}\left(\mathbf{w}_{L}\right) & \text { if } & x<0 \\
\mathbf{u}_{R}=\mathcal{U}\left(\mathbf{w}_{R}\right) & \text { if } & x>0
\end{array}\right.
\end{aligned}
$$

where $\tilde{\mathbf{u}}:=\tilde{\mathbf{u}}\left(\mathbf{u}_{L}, \mathbf{u}_{R}\right)$ represents any averaging of the variable $\mathbf{u}_{L}$ and $\mathbf{u}_{R}$. One of the aim of the present paper is to discuss these averagings and to propose relevant choice to preserve physical assumptions. With some abuse in the notations, we will set $B(\tilde{\mathbf{u}}):=\tilde{B}\left(\mathbf{u}_{L}, \mathbf{u}_{R}\right)$.

Since the above problem is linear, the exact solution $\mathbf{u}^{*}\left(x / t ; \mathbf{u}_{L}, \mathbf{u}_{R}\right)$ is easily obtained. A numerical flux function is then defined as follows:

$$
\mathbf{f}^{*}\left(\mathbf{w}_{L}, \mathbf{w}_{R}\right)=\mathbf{f}\left(\mathcal{W}\left(\mathbf{u}^{*}\left(0 ; \mathcal{U}\left(\mathbf{w}_{L}\right), \mathcal{U}\left(\mathbf{w}_{R}\right)\right)\right)\right.
$$

Hence the explicit form of the finite volume VFRoe scheme is given by:

$\mathbf{w}_{i}^{n+1}=\mathbf{w}_{i}^{n}-\frac{\Delta t}{\Delta x}\left(\mathbf{f}^{*}\left(\mathcal{W}\left(\mathbf{u}^{*}\left(0 ; \mathcal{U}\left(\mathbf{w}_{i}^{n}\right), \mathcal{U}\left(\mathbf{w}_{i+1}^{n}\right)\right)\right)-\mathbf{f}^{*}\left(\mathcal{W}\left(\mathbf{u}^{*}\left(0 ; \mathcal{U}\left(\mathbf{w}_{i-1}^{n}\right), \mathcal{U}\left(\mathbf{w}_{i}^{n}\right)\right)\right)\right)\right.\right.$. 
The numerical flux function is obviously consistent with the exact flux function since we have $\mathbf{u}^{*}(0, \mathbf{u}, \mathbf{u})=\mathbf{u}$ for all $\mathbf{u}$ and then we get

$$
\mathbf{f}^{*}(\mathbf{w}, \mathbf{w})=\mathbf{f}(\mathcal{W}(\mathcal{U}(\mathbf{w})))=\mathbf{f}(\mathbf{w}) .
$$

This leads to a conservation scheme (2.5) for any linearization matrix $\tilde{B}\left(\mathbf{u}_{L}, \mathbf{u}_{R}\right)$.

The solution of the linearized Riemann problem (2.2) is defined everywhere except along $x / t=\tilde{\lambda}_{k}$ with the following relations:

$$
\begin{aligned}
\mathbf{u}^{*}\left(x / t, \mathbf{u}_{L}, \mathbf{u}_{R}\right) & =\mathbf{u}_{L}+\sum_{\frac{x}{t}>\tilde{\lambda}_{k}}\left({ }^{t} \tilde{\mathbf{l}}_{k} \cdot[\mathbf{u}]_{L}^{R}\right) \tilde{\mathbf{r}}_{k}, \\
& =\mathbf{u}_{R}-\sum_{\frac{x}{t}<\tilde{\lambda}_{k}}\left({ }^{t} \tilde{\mathbf{l}}_{k} \cdot[\mathbf{u}]_{L}^{R}\right) \tilde{\mathbf{r}}_{k},
\end{aligned}
$$

with $[\mathbf{u}]_{L}^{R}=\mathbf{u}_{R}-\mathbf{u}_{L}$, where we have set $\tilde{\mathbf{l}}_{k}, \tilde{\lambda}_{k}$ and $\tilde{\mathbf{r}}_{k}, k=1, \ldots, p$ respectively as the left eigenvectors, eigenvalues and right eigenvectors of $B(\tilde{\mathbf{u}})$.

We recall that the basic VFRoe scheme was first introduced in [35] with the more simple choice $\mathcal{U}(\mathbf{w})=\mathbf{w}$ and thus $B(\tilde{\mathbf{u}})=A(\tilde{\mathbf{w}})$. In particular, the stability of the VFRoe scheme in the scalar case is studied in [35]. Various changes of variables for the Euler equations were examined in [8, 9] and in [13], [14] for the shallow water equations.

Now, we specify the scheme when considering the shallow water equations (1.5). The solution of the associated Riemann problem, in the absence of dry areas, is composed of three distinct constant states separated by two genuinely non-linear fields, of speeds $u-c$ and $u+c$ respectively, where the celerity is usually defined by $c=\sqrt{g h}$. Motivated by the form of the Riemann invariant associated with the wave speeds, and far away from the dry areas $(h>0)$, we consider herein the change of variable $\mathcal{U}(\mathbf{w})={ }^{t}(2 c, u)$. The system (1.5) may be written related to $\mathbf{u}$ in the following non conservation form:

$$
\left\{\begin{array}{l}
\partial_{t}(2 c)+u \partial_{x}(2 c)+c \partial_{x} u=0 \\
\partial_{t} u+c \partial_{x}(2 c)+u \partial_{x} u=0
\end{array}\right.
$$

The Jacobian matrix $B(\mathbf{u})$ becomes thus:

$$
B(\mathbf{u})=\left(\begin{array}{ll}
u & c \\
c & u
\end{array}\right)
$$

In [14], the averaged state $\left(\mathbf{u}_{L}+\mathbf{u}_{R}\right) / 2$ is proposed for the linearization involved in $B(\tilde{\mathbf{u}})$ and thus for the characterization of $\tilde{u}$ and $\tilde{c}$. In the present work, the choice of the linearization stays free and it will be specified later on. Independently on the definition of $\tilde{u}$ and $\tilde{c}$, we obtain the eigenvalues $\lambda^{ \pm}=\tilde{u} \pm \tilde{c}$. The exact solution of the linearized Riemann problem (2.2) is easily computed to obtain

$$
\mathcal{W}\left(\mathbf{u}^{*}\left(0 ; \mathcal{U}\left(\mathbf{w}_{i}^{n}\right), \mathcal{U}\left(\mathbf{w}_{i+1}^{n}\right)\right)\right)= \begin{cases}\mathbf{w}_{i}^{n} & \text { if }\left(\lambda^{-}\right)_{i+\frac{1}{2}}>0 \\ \mathcal{W}\left(\mathbf{u}_{i+\frac{1}{2}}\right) & \text { if }\left(\lambda^{-}\right)_{i+\frac{1}{2}}<0<\left(\lambda^{+}\right)_{i+\frac{1}{2}} \\ \mathbf{w}_{i+1}^{n} & \text { if }\left(\lambda^{+}\right)_{i+\frac{1}{2}}<0\end{cases}
$$

where $\left(\lambda^{ \pm}\right)_{i+\frac{1}{2}}=\tilde{u}_{i+\frac{1}{2}} \pm \tilde{c}_{i+\frac{1}{2}}$ according to the choice of the adopted linearization of $\tilde{u}$ and $\tilde{c}$. Here, we have set $\mathbf{u}_{i+\frac{1}{2}}={ }^{t}\left(2 c_{i+\frac{1}{2}}, u_{i+\frac{1}{2}}\right)$ where

$$
c_{i+\frac{1}{2}}=\frac{1}{2}\left(c_{i}+c_{i+1}\right)-\frac{1}{4}\left(u_{i+1}-u_{i}\right), \quad u_{i+\frac{1}{2}}=\frac{1}{2}\left(u_{i}+u_{i+1}\right)-\left(c_{i+1}-c_{i}\right) .
$$


This concludes the presentation of the considered VFRoe scheme. Lastly, we emphasize that actually, the definition of $\tilde{u}$ and $\tilde{c}$ only influences the upwind definition to use.

3. A relaxation approach. Motivated by the pioneer work of Jin-Xin [26] (see also $[11,32]$ ), we propose to introduce a relevant relaxation scheme to obtain a suitable interpretation of the VFRoe scheme (2.5)-(2.9). We approximate the weak solutions of the system (1.5) by the weak solutions of a suitable first order system with singular perturbations: the relaxation model. According to the work of Coquel-Perthame [12] or Bouchut [7] (see also [2,3]), we propose a relaxation model which preserves most of the nonlinearities of the initial system. The resulting numerical scheme, based on the relaxation model, will be seen to coincide with the VFRoe method.

3.1. The relaxation model. Following the work of Suliciu [39, 40] (see also $[2,3,7,12])$, we suggest substituting the celerity $c$ and velocity $u$ by the approximation $\Sigma$ and $U$. These two new variables are governed by

$$
\left\{\begin{array}{l}
\partial_{t} \Sigma+\bar{u} \partial_{x} \Sigma+\frac{\bar{c}}{2} \partial_{x} U=\mu(c-\Sigma) \\
\partial_{t} U+2 \bar{c} \partial_{x} \Sigma+\bar{u} \partial_{x} U=\mu(u-U)
\end{array}\right.
$$

where $\bar{c}$ and $\bar{u}$ are relaxation parameters to be defined while $\mu$ is a parameter intended to tend to infinity. The following first order system with singular perturbations:

$$
\left\{\begin{array}{l}
\partial_{t} h+\partial_{x}\left(\frac{\Sigma^{2}}{g} U\right)=0, \quad t>0, x \in \mathbb{R}, \\
\partial_{t}(h u)+\partial_{x}\left(\frac{\Sigma^{2}}{2 g}\left(2 U^{2}+\Sigma^{2}\right)\right)=0 \\
\partial_{t} \Sigma+\bar{u} \partial_{x} \Sigma+\frac{\bar{c}}{2} \partial_{x} U=\mu(c-\Sigma), \\
\partial_{t} U+2 \bar{c} \partial_{x} \Sigma+\bar{u} \partial_{x} U=\mu(u-U),
\end{array}\right.
$$

is considered to approximate the weak solutions of (1.5). Now, at least formally, in the limit of $\mu$ to infinity, we recover the initial system (1.5). This limit will be referred to as the equilibrium limit, defined by $\Sigma=c$ and $U=u$. The conservation of the water height $h$ and the discharge $h u$ in (3.1) then gives those of (1.5). In the following, we establish that the scheme obtained from the relaxation model (3.1) is relevant, in a sense to be precised, to approximate solutions involving dry areas.

For the sake of simplicity in the notations, let us introduce the following abstract form of the relaxation system (3.1):

$$
\partial_{t} \mathbf{W}+\partial_{x} \mathbf{F}(\mathbf{W})=\mu \mathbf{R}(\mathbf{W})
$$

where we have set $\mathbf{W}={ }^{t}(h, h u, \Sigma, U)$ defined over the following convex set:

$$
\mathcal{V}=\left\{\mathbf{W} \in \mathbb{R}^{4} ; h \geq 0\right\} .
$$

One of the main interest of the relaxation model (3.1) stays in the linear degeneracy property satisfied by all the fields. As a consequence, the Riemann problem turns out to be easy to solve. The next statement is devoted to solve the Riemann problem.

Lemma 3.1. Let be given $\bar{c}>0$ and $\bar{u} \in \mathbb{R}$ such that $\bar{u} \pm \bar{c} \neq 0$. Assume $\mu=0$. The first order system $(3.1)_{\mu=0}$ is hyperbolic for all $\mathbf{W} \in \mathcal{V}$. It admits $\lambda_{1}^{0}=\lambda_{2}^{0}=0$ and $\lambda^{ \pm}=\bar{u} \pm \bar{c}$ as eigenvalues and the associated fields are linearly degenerated. 
Let $\mathbf{W}_{L}$ and $\mathbf{W}_{R}$ be constant states in $\mathcal{V}$ and define

$$
\mathbf{W}_{0}(x)= \begin{cases}\mathbf{W}_{L} & \text { if } x<0 \\ \mathbf{W}_{R} & \text { if } x>0\end{cases}
$$

the initial data of the Riemann problem for the system $(3.1)_{\mu=0}$. Let us set

$$
\begin{aligned}
\Sigma^{*} & =\frac{\Sigma_{L}+\Sigma_{R}}{2}-\frac{1}{4}\left(U_{R}-U_{L}\right), \\
U^{*} & =\frac{U_{L}+U_{R}}{2}-\left(\Sigma_{R}-\Sigma_{L}\right) .
\end{aligned}
$$

Then the weak solution of the system $(3.1)_{\mu=0}$ and for the initial data (3.3) is given by

1. If $\lambda^{-}<0<\lambda^{+}$:

$$
\mathbf{W}(x, t)=\left\{\begin{array}{ccccc}
\mathbf{W}_{L} & \text { if } & & x / t & <\lambda^{-} \\
\mathbf{W}_{L}^{*} & \text { if } & \lambda^{-}< & x / t & <0, \\
\mathbf{W}_{R}^{*} & \text { if } & 0< & x / t & <\lambda^{+}, \\
\mathbf{W}_{R} & \text { if } & \lambda^{+}< & x / t &
\end{array}\right.
$$

where

$$
\mathbf{W}_{L}^{*}={ }^{t}\left(h_{L}^{*}, q_{L}^{*}, \Sigma^{*}, U^{*}\right), \quad \mathbf{W}_{R}^{*}={ }^{t}\left(h_{R}^{*}, q_{R}^{*}, \Sigma^{*}, U^{*}\right),
$$

and the values of $\left(h_{L}^{*}, q_{L}^{*}\right)$ and $\left(h_{R}^{*}, q_{R}^{*}\right)$ are given by

$h_{L}^{*}=h_{L}+\frac{\Sigma^{* 2} U^{*}-\Sigma_{L}^{2} U_{L}}{g \lambda^{-}}, \quad q_{L}^{*}=(h u)_{L}-\frac{\mathcal{I}\left(\Sigma^{*}, U^{*}\right)-\mathcal{I}\left(\Sigma_{L}, U_{L}\right)}{\lambda^{-}}$,

$h_{R}^{*}=h_{R}+\frac{\Sigma^{* 2} U^{*}-\Sigma_{R}^{2} U_{R}}{g \lambda^{+}}, \quad q_{R}^{*}=(h u)_{R}-\frac{\mathcal{I}\left(\Sigma^{*}, U^{*}\right)-\mathcal{I}\left(\Sigma_{R}, U_{R}\right)}{\lambda^{+}}$,

where the function $\mathcal{I}: \mathbb{R}^{2} \rightarrow \mathbb{R}$ is defined by

$$
\mathcal{I}(\Sigma, U)=\frac{\Sigma^{2}}{2 g}\left(\Sigma^{2}+2 U^{2}\right)
$$

2. If $0<\lambda^{-}<\lambda^{+}$:

$$
\mathbf{W}(x, t)=\left\{\begin{array}{llll}
\mathbf{W}_{L} & \text { if } & x / t<0 \\
\mathbf{W}_{L}^{*} & \text { if } \quad 0<x / t<\lambda^{-}, \\
\mathbf{W}_{R}^{*} & \text { if } \lambda^{-}<x / t<\lambda^{+}, \\
\mathbf{W}_{R} & \text { if } \lambda^{+}<x / t,
\end{array}\right.
$$

where

$$
\mathbf{W}_{L}^{*}={ }^{t}\left(h_{L}^{*}, q_{L}^{*}, \Sigma_{L}, U_{L}\right), \quad \mathbf{W}_{R}^{*}={ }^{t}\left(h_{R}^{*}, q_{R}^{*}, \Sigma^{*}, U^{*}\right),
$$

and the values of $\left(h_{L}^{*}, q_{L}^{*}\right)$ and $\left(h_{R}^{*}, q_{R}^{*}\right)$ are given by

$h_{L}^{*}=h_{R}^{*}+\frac{\Sigma_{L}^{2} U_{L}-\Sigma^{* 2} U^{*}}{g \lambda^{-}}, \quad q_{L}^{*}=q_{R}^{*}-\frac{\mathcal{I}\left(\Sigma_{L}, U_{L}\right)-\mathcal{I}\left(\Sigma^{*}, U^{*}\right)}{\lambda^{-}}$,

$h_{R}^{*}=h_{R}+\frac{\Sigma^{* 2} U^{*}-\Sigma_{R}^{2} U_{R}}{g \lambda^{+}}, \quad q_{R}^{*}=(h u)_{R}-\frac{\mathcal{I}\left(\Sigma^{*}, U^{*}\right)-\mathcal{I}\left(\Sigma_{R}, U_{R}\right)}{\lambda^{+}}$,

where the function $\mathcal{I}$ is defined by (3.8). 
3. If $\lambda^{-}<\lambda^{+}<0$ :

$$
\mathbf{W}(x, t)=\left\{\begin{array}{clll}
\mathbf{W}_{L} & \text { if } & & x / t<\lambda^{-} \\
\mathbf{W}_{L}^{*} & \text { if } \lambda^{-}<x / t<\lambda^{+}, \\
\mathbf{W}_{R}^{*} & \text { if } \lambda^{+}<x / t<0, \\
\mathbf{W}_{R} & \text { if } \quad 0<x / t,
\end{array}\right.
$$

where

$$
\mathbf{W}_{L}^{*}={ }^{t}\left(h_{L}^{*}, q_{L}^{*}, \Sigma^{*}, U^{*}\right), \quad \mathbf{W}_{R}^{*}={ }^{t}\left(h_{R}^{*}, q_{R}^{*}, \Sigma_{R}, U_{R}\right)
$$

and the values of $\left(h_{L}^{*}, q_{L}^{*}\right)$ and $\left(h_{R}^{*}, q_{R}^{*}\right)$ are given by

$$
\begin{aligned}
& h_{L}^{*}=h_{L}+\frac{\Sigma^{* 2} U^{*}-\Sigma_{L}^{2} U_{L}}{g \lambda^{-}}, \quad q_{L}^{*}=(h u)_{L}-\frac{\mathcal{I}\left(\Sigma^{*}, U^{*}\right)-\mathcal{I}\left(\Sigma_{L}, U_{L}\right)}{\lambda^{-}}, \\
& h_{R}^{*}=h_{L}^{*}+\frac{\Sigma_{R}^{2} U_{R}-\Sigma^{* 2} U^{*}}{g \lambda^{+}}, \quad q_{R}^{*}=q_{L}^{*}-\frac{\mathcal{I}\left(\Sigma_{R}, U_{R}\right)-\mathcal{I}\left(\Sigma^{*}, U^{*}\right)}{\lambda^{+}},
\end{aligned}
$$

where the function $\mathcal{I}$ is, once again, defined by (3.8).

Let us note from now on that the intermediate values $\Sigma^{*}$ and $U^{*}$ remain free from the parameters $\bar{c}$ and $\bar{u}$. This remark will be crucial in the sequel when establishing relevant relationship between the relaxation scheme and the VFRoe scheme. Indeed, we will see that the relaxation numerical flux function solely depends on the pair $\left(\Sigma^{*}, U^{*}\right)$, but for the definition (3.4)-(3.5) this function will coincide with the VFRoe numerical flux function given by (2.5)-(2.9). This remark will be obtained independently from the precise definition of the pair $(\bar{u}, \bar{c})$.

Proof. Considering the algebra of the system (3.2), the flux function Jacobian matrix is given by

$$
\nabla_{\mathbf{W}} \mathbf{F}(\mathbf{W})=\left(\begin{array}{llll}
0 & 0 & 2 \Sigma U / g & \Sigma^{2} / g \\
0 & 0 & \Sigma\left(U^{2}+\Sigma^{2}\right) / g & 2 \Sigma^{2} U / g \\
0 & 0 & \bar{u} & \bar{c} / 2 \\
0 & 0 & 2 \bar{c} & \bar{u}
\end{array}\right)
$$

Easy calculations ensure that the vectors given by $\mathbf{r}_{1}^{0}={ }^{t}(1,0,0,0), \mathbf{r}_{2}^{0}={ }^{t}(0,1,0,0)$ and

$$
\mathbf{r}^{ \pm}=\left(\begin{array}{l}
2 \Sigma U / g \pm 2 \Sigma^{2} / g \\
2 \Sigma\left(U^{2}+\Sigma^{2}\right) / g \pm 4 \Sigma^{2} U / g \\
\bar{u} \pm \bar{c} \\
\pm 2(\bar{u} \pm \bar{c})
\end{array}\right)
$$

are the right eigenvectors respectively associated to the eigenvalues $\lambda_{1}^{0}=\lambda_{2}^{0}=0$ and $\lambda^{ \pm}=\bar{u} \pm \bar{c}$. In addition, it is easy to see that all the fields are linearly degenerated.

The Riemann solution is made of four constant states $\mathbf{W}_{L}, \mathbf{W}_{L}^{*}, \mathbf{W}_{R}^{*}$, and $\mathbf{W}_{R}$, separated by three contact discontinuities, a stationary and two propagating at velocity $\lambda^{ \pm}$. The above third developed cases only aim at dealing with the location of the wave velocities $\lambda^{-}$and $\lambda^{+}$with respect to the stationary discontinuity. We recall that across the $j$ th-contact discontinuity, the Riemann invariants associated with the $j$ th-eigenvector are continuous. These Riemann invariants denoted $\phi_{j}$ are defined by 
$\nabla \phi_{j} \cdot \mathbf{r}_{j}=0$ where $\mathbf{r}_{j}$ is the $j$ th right eigenvector introduced above. After straightforward computations, we obtain the following Riemann invariants, defined field by field:

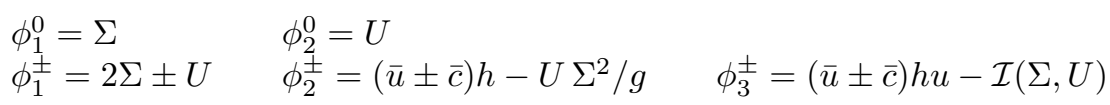

Now, considering the case $\lambda^{-}<0<\lambda^{+}$and exploiting the continuity of these invariants across the associated contact discontinuity, we obtain the following system of equations:

$$
\begin{aligned}
& \left\{\begin{array}{l}
\Sigma_{L}^{*}=\Sigma_{R}^{*} \\
U_{L}^{*}=U_{R}^{*}
\end{array}\right. \\
& \left\{\begin{array}{l}
U_{L}+2 \Sigma_{L}=U^{*}+2 \Sigma^{*} \\
\lambda^{-} h_{L}-\frac{U_{L} \Sigma_{L}^{2}}{g}=\lambda^{-} h_{L}^{*}-\frac{U^{*}\left(\Sigma^{*}\right)^{2}}{g} \\
\lambda^{-}(h u)_{L}-\mathcal{I}\left(\Sigma_{L}, U_{L}\right)=\lambda^{-} q_{L}^{*}-\mathcal{I}\left(\Sigma^{*}, U^{*}\right)
\end{array}\right. \\
& \left\{\begin{array}{l}
U^{*}-2 \Sigma^{*}=U_{R}-2 \Sigma_{R} \\
\lambda^{+} h_{R}^{*}-\frac{U^{*} \Sigma_{L}^{2}}{g}=\lambda^{+} h_{R}-\frac{U_{R} \Sigma_{R}^{2}}{g} \\
\lambda^{+}(h u)_{R}-\mathcal{I}\left(\Sigma_{R}, U_{R}\right)=\lambda^{+} q_{R}^{*}-\mathcal{I}\left(\Sigma^{*}, U^{*}\right)
\end{array}\right.
\end{aligned}
$$

The unique solution of this system is given by (3.4)-(3.7). The two remaining cases are developed following the same lines. The proof is thus completed.

This analysis of the Riemann problem solutions can be supplemented by a study of the non-negativity of the water depth $h$, establishing that $h_{L, R}^{*} \geq 0$ as soon as $h_{L, R} \geq 0$. At the moment, let us focus our attention on $h_{L}^{*}$; the same approach will be used considering $h_{R}^{*}$.

Since $\Sigma^{*}$ and $U^{*}$ do not depend on the eigenvalues $\lambda^{ \pm}$, while $\lambda^{ \pm}$does not depend on the unknowns but just on the fixed parameters $\bar{u}$ and $\bar{c}$, with $h_{L}>0$ and $h_{R}>0$ it is clear that $h_{L}^{*}$ remains non-negative as soon as $\left|\lambda^{ \pm}\right|$is larger enough. Now, assume $h_{L}=0$ and $h_{R}>0$, to write with $U_{L}=0$ :

$$
h_{L}^{*}= \begin{cases}\frac{1}{\lambda^{-} g}\left(\Sigma^{*}\right)^{2} U^{*} & \text { if } \lambda^{-}<0 \\ h_{R}+\frac{1}{\lambda^{+} g}\left(\left(\Sigma^{*}\right)^{2} U^{*}-\Sigma_{R}^{2} U_{R}\right)-\frac{1}{\lambda^{-} g}\left(\Sigma^{*}\right)^{2} U^{*} & \text { if } \lambda^{-}>0\end{cases}
$$

Two cases must be distinguished. If $U^{*}<0$, we have just to set $\lambda^{-}<0$. Reversely, if we have $U^{*}>0$, the parameters $\bar{u}$ and $\bar{c}$ must be chosen to enforce $0<\lambda^{-}<\lambda^{+}$large enough to satisfy $h_{L}^{*}>0$. Finally, in the case of $h_{L}=h_{R}=0$, with the convention $U_{L}=U_{R}=0$, we obtain $h_{L}^{*}=0$. Involving the same analysis with $h_{R}^{*}$, we have easily established the following result:

LemMa 3.2. Assume $h_{L} \geq 0$ and $h_{R} \geq 0$ with the convention $U_{L}=0$ if $h_{L}=0$ and $U_{R}=0$ if $h_{R}=0$. Then there exist suitable parameters $\bar{u}$ and $\bar{c}$ such that the functions $h_{L}^{*}$ and $h_{R}^{*}$, defined in Lemma 3.1, are non-negative.

In order to help the reader in the full determination of the relaxation model, and thus in the characterization of the relaxation scheme, we underline that the relevant definition of the eigenvalues $\lambda^{ \pm}$coincides with the following choice of the relaxation parameters $(\bar{u}, \bar{c})$ :

$$
\bar{u}=\frac{\lambda^{+}+\lambda^{-}}{2} \quad \text { and } \quad \bar{c}=\frac{\lambda^{+}-\lambda^{-}}{2}
$$


¿From a practical point of view, one wants to define $\bar{u}=\left(u_{L}+u_{R}\right) / 2$ and $\bar{c}=\left(c_{L}+\right.$ $\left.c_{R}\right) / 2$ (see [14]). As an example, such a choice can be considered and locally modified as soon as the non-negativity of $h_{L}^{*}$ or $h_{R}^{*}$ is violated. Of course this local correction of $\bar{u}$ and $\bar{c}$ is done according to Lemma 3.2. It is clear that many other choices can be suggested and the numerical consequences resulting from the considered definition of $\bar{u}$ and $\bar{c}$ will be detailed in the next section. Under a more general framework, we can set $\bar{u}:=\bar{u}\left(\mathbf{W}_{L}, \mathbf{W}_{R}\right)$ and $\bar{c}:=\bar{c}\left(\mathbf{W}_{L}, \mathbf{W}_{R}\right)$ which reads $\bar{u}:=\bar{u}\left(\mathbf{w}_{L}, \mathbf{w}_{R}\right)$ and $\bar{c}:=\bar{c}\left(\mathbf{w}_{L}, \mathbf{w}_{R}\right)$ as soon as the states $\mathbf{W}_{L}$ and $\mathbf{W}_{R}$ are assumed to satisfy the equilibrium, defined by $U=u$ and $\Sigma=\sqrt{g h}$.

3.2. The relaxation solver. For the sake of completeness, we briefly recall the numerical relaxation procedure to approximate weak solution of (1.5), which is usual in the framework of the relaxation scheme (see Jin-Xin [26], but also Coquel-Perthame [12], Baudin et al.[2], Bouchut [7] or Berthon [3]).

We consider the same mesh notations as introduced in section 2 . To approximate the solution at time $t^{n+1}=t^{n}+\Delta t$, a splitting technique is adopted. In a first step, we solve the relaxation model (3.2) omitting the relaxation source terms which are considered in a second step. As usual, we assume that a piecewise constant approximate equilibrium solution $\mathbf{w}^{h}\left(x, t^{n}\right) \in \Omega$ is known at time $t^{n}$, defined by

$$
\mathbf{w}^{h}\left(x, t^{n}\right)=\mathbf{w}_{i}^{n}={ }^{t}\left(h_{i}^{n},(h u)_{i}^{n}\right), \quad x \in\left(x_{i-\frac{1}{2}}, x_{i+\frac{1}{2}}\right) .
$$

At the initial time, we set

$$
\mathbf{w}_{i}^{0}=\frac{1}{\Delta x} \int_{x_{i-\frac{1}{2}}}^{x_{i+\frac{1}{2}}} \mathbf{w}(x, 0) d x .
$$

During the first step, we propose to evolve in time a relevant approximation of the relaxation model (3.2). To access such an issue, we introduce $\mathbf{W}^{h} \in \mathcal{V}$ such that for all $0<t<\Delta t$, the function $\mathbf{W}^{h}\left(x, t^{n}+t\right)$ is the weak solution of the Cauchy problem for the relaxation system $(3.2)_{\mu=0}$ :

$$
\partial_{t} \mathbf{W}+\partial_{x} \mathbf{F}(\mathbf{W})=0
$$

supplemented by the following initial equilibrium data:

$$
\begin{aligned}
\mathbf{W}^{h}\left(x, t^{n}\right) & =\mathbf{W}_{i}^{n} \\
& ={ }^{t}\left(h_{i}^{n},(h u)_{i}^{n}, \Sigma_{i}^{n}, U_{i}^{n}\right), \quad x \in\left(x_{i-\frac{1}{2}}, x_{i+\frac{1}{2}}\right),
\end{aligned}
$$

where the equilibrium state is defined by $\Sigma_{i}^{n}=\sqrt{g h_{i}^{n}}$ and $U_{i}^{n}=(h u)_{i}^{n} / h_{i}^{n}$. Under the CFL like condition

$$
\frac{\Delta t}{\Delta x} \max _{i \in \mathbb{Z}}\left(\left|\lambda_{i+\frac{1}{2}}^{-}\right|,\left|\lambda_{i+\frac{1}{2}}^{+}\right|\right) \leq \frac{1}{2},
$$

the solution $\mathbf{W}^{h}$ at the time $t^{n}+\Delta t$ is made of the juxtaposition of the non-interacting Riemann problem solution set at the cell interfaces $x_{i+\frac{1}{2}}$ for $i \in \mathbb{Z}$. Next, the projection of this solution on the piecewise constant functions reads:

$$
\mathbf{W}_{i}^{n+1,-}=\frac{1}{\Delta x} \int_{x_{i-\frac{1}{2}}}^{x_{i+\frac{1}{2}}} \mathbf{W}^{h}\left(x, t^{n}+\Delta t\right) d x .
$$


A local definition of the parameter $\bar{u}$ and $\bar{c}$ at each interface $x_{i+\frac{1}{2}}$ is considered (for instance, see [14]). At each interface $x_{i+\frac{1}{2}}$, we choose $\mathbf{W}_{L}=\mathbf{W}_{i}^{n}$ and $\mathbf{W}_{R}=\mathbf{W}_{i+1}^{n}$ to define the parameters $\bar{u}_{i+\frac{1}{2}}:=\bar{u}\left(\mathbf{w}_{i}^{n}, \mathbf{w}_{i+1}^{n}\right)$ and $\bar{c}_{i+\frac{1}{2}}:=\bar{c}\left(\mathbf{w}_{i}^{n}, \mathbf{w}_{i+1}^{n}\right)$ according to the non-negativity condition stated Lemma 3.2. Assuming the CFL restriction (3.19), the relaxation parameters may vary from one interface to another. For convenience in the sequel and to emphasize the admissible local choice of the parameters, we rewrite $\mathbf{W}_{i}^{n+1,-}$ arguing the well-known formalism introduced by Harten, Lax and van Leer [21]:

$$
\mathbf{W}_{i}^{n+1,-}=\frac{1}{2}\left(\overline{\mathbf{W}}_{R}\left(\mathbf{W}_{i-1}^{n}, \mathbf{W}_{i}^{n}\right)+\overline{\mathbf{W}}_{L}\left(\mathbf{W}_{i}^{n}, \mathbf{W}_{i+1}^{n}\right)\right)
$$

where

$$
\begin{aligned}
\overline{\mathbf{W}}_{L}\left(\mathbf{W}_{L}, \mathbf{W}_{R}\right) & =\frac{2 \Delta t}{\Delta x} \int_{-\frac{\Delta x}{2 \Delta t}}^{0} \mathbf{W}_{r}\left(\xi ; \mathbf{W}_{L}, \mathbf{W}_{R}\right) d \xi \\
& =\mathbf{W}_{L}-\frac{2 \Delta t}{\Delta x}\left(\mathbf{F}\left(\mathbf{W}_{r}\left(0 ; \mathbf{W}_{L}, \mathbf{W}_{R}\right)\right)-\mathbf{F}\left(\mathbf{W}_{L}\right)\right),
\end{aligned}
$$

and

$$
\begin{aligned}
\overline{\mathbf{W}}_{R}\left(\mathbf{W}_{L}, \mathbf{W}_{R}\right) & =\frac{2 \Delta t}{\Delta x} \int_{0}^{\frac{\Delta x}{2 \Delta t}} \mathbf{W}_{r}\left(\xi ; \mathbf{W}_{L}, \mathbf{W}_{R}\right) d \xi \\
& =\mathbf{W}_{R}-\frac{2 \Delta t}{\Delta x}\left(\mathbf{F}\left(\mathbf{W}_{R}\right)-\mathbf{F}\left(\mathbf{W}_{r}\left(0 ; \mathbf{W}_{L}, \mathbf{W}_{R}\right)\right)\right)
\end{aligned}
$$

The function $\mathbf{W}_{r}\left(. ; \mathbf{W}_{L}, \mathbf{W}_{R}\right)$ denotes the solution of the Riemann problem for (3.18) where the initial data is prescribed by (3.3).

As soon as $\mathbf{W}_{L}:=\mathbf{W}\left(\mathbf{w}_{L}\right)$ and $\mathbf{W}_{R}:=\mathbf{W}\left(\mathbf{w}_{R}\right)$ are defined from the equilibrium states $\mathbf{w}_{L}$ and $\mathbf{w}_{R}$, it is crucial to notice from now on the following identities:

$$
\begin{aligned}
& \left.\mathbf{F}\left(\mathbf{W}_{L}\right)\right|_{[h, h u]}=\mathbf{f}\left(\mathbf{w}_{L}\right), \\
& \left.\mathbf{F}\left(\mathbf{W}_{R}\right)\right|_{[h, h u]}=\mathbf{f}\left(\mathbf{w}_{R}\right),
\end{aligned}
$$

where the notation $\left.\mathbf{F}()\right|_{.[h, h u]}$ denotes the restriction of $\mathbf{F}$ to the component $(h, h u)$.

The second step of the scheme is devoted to the relaxation procedure. At time $t=t^{n}+\Delta t$, we define the updated approximate equilibrium solution $\mathbf{w}^{n+1}(x)$ as follows:

$$
\mathbf{w}^{n+1}(x)={ }^{t}\left(h_{i}^{n+1,-},(h u)_{i}^{n+1,-}\right), \quad x \in\left(x_{i-\frac{1}{2}}, x_{i+\frac{1}{2}}\right),
$$

and we set $\Sigma_{i}^{n+1}=\sqrt{g h_{i}^{n+1}}$ and $U_{i}^{n+1}=(h u)_{i}^{n+1} / h_{i}^{n+1}$.

In fact, this second step amounts solving the system

$$
\partial_{t} \mathbf{W}=\mu \mathbf{R}(\mathbf{W})
$$

with the piecewise constant approximation $\mathbf{W}_{i}^{n+1,-}$ as initial data while $\mu$ tends to infinity. The derivation of the relaxation scheme is thus achieved.

In fact, the numerical relaxation flux function, we have just derived, exactly coincide with the VFRoe numerical flux function given by (2.5)-(2.9). Involving the 
classical framework of the finite volume method, the described relaxation scheme summarizes as follows:

$$
\mathbf{w}_{i}^{n+1}=\mathbf{w}_{i}^{n}-\frac{\Delta t}{\Delta x}\left(\mathbf{f}_{i+\frac{1}{2}}^{n}-\mathbf{f}_{i-\frac{1}{2}}^{n}\right)
$$

where the numerical flux function is defined by

$$
\begin{aligned}
\mathbf{f}_{i+\frac{1}{2}}^{n} & =\mathbf{f}\left(\mathbf{w}_{i}^{n}, \mathbf{w}_{i+1}^{n}\right), \\
& =\left.\mathbf{F}\left(\mathbf{W}_{r}\left(0 ; \mathbf{W}\left(\mathbf{w}_{i}^{n}\right), \mathbf{W}\left(\mathbf{w}_{i+1}^{n}\right)\right)\right)\right|_{[h, h u]},
\end{aligned}
$$

with $\mathbf{W}_{i}^{n}=\mathbf{W}\left(\mathbf{w}_{i}^{n}\right)$ defined according to the equilibrium, i.e. $\Sigma_{i}^{n}=c_{i}^{n}$ and $U_{i}^{n}=u_{i}^{n}$.

Now, it is clear that both relaxation scheme (3.24)-(3.25) and VFRoe scheme (2.5)-(2.9) involve the same numerical flux function in the form:

$$
\mathbf{f}\left(\mathbf{w}_{L}, \mathbf{w}_{R}\right)=\left(\begin{array}{l}
\left(c^{*}\right)^{2} u^{*} / g \\
\left(c^{*}\right)^{2}\left(2\left(u^{*}\right)^{2}+\left(c^{*}\right)^{2}\right) /(2 g)
\end{array}\right),
$$

where

$$
\begin{aligned}
& c^{*}= \begin{cases}c_{L} & \text { if } \lambda^{-}>0, \\
\frac{c_{L}+c_{R}}{2}-\frac{1}{4}\left(u_{R}-u_{L}\right) & \text { if } \lambda^{-}<0<\lambda^{+}, \\
c_{R} & \text { if } \lambda^{+}<0,\end{cases} \\
& u^{*}= \begin{cases}u_{L} & \text { if } \lambda^{-}>0, \\
\frac{u_{L}+u_{R}}{2}-\left(c_{R}-c_{L}\right) & \text { if } \lambda^{-}<0<\lambda^{+}, \\
u_{R} & \text { if } \lambda^{+}<0 .\end{cases}
\end{aligned}
$$

In fact, the two schemes may differ in the evaluation of the eigenvalues $\lambda^{ \pm}$. In this sense, the VFRoe scheme (2.5)-(2.9) is closed when enforcing the linearization $\tilde{u}=\bar{u}$ and $\tilde{c}=\bar{c}$. We have thus proposed a relaxation interpretation of the well-known VFRoe scheme. We conclude establishing the following expected robustness result:

TheOREm 3.3. Assume that $\mathbf{w}_{i}^{n} \in \Omega$ for all $i \in \mathbb{Z}$ and assume that the eigenvalues $\lambda_{i+\frac{1}{2}}^{ \pm}$are evaluated according to the depth non-negativity Lemma 3.2. Under the CFL condition (3.19), the relaxation scheme (3.24)-(3.25), or equivalently the VFRoe scheme (2.5)-(2.9), preserves the non-negativity of $h: h_{i}^{n+1} \geq 0$ for all $i \in \mathbb{Z}$. In addition, the scheme does not involve blow-up when dry areas are encountered.

Proof. Since Lemma 3.2 is satisfied, the intermediate states, involved in the Riemann problem solution at each interface, preserve the non-negativity of the depth. Then $h^{h}\left(x, t^{n}+\Delta t\right) \geq 0$ for all $x \in \mathbb{R}$, while we have

$$
h_{i}^{n+1}=\frac{1}{\Delta x} \int_{x_{i-\frac{1}{2}}}^{x_{i+\frac{1}{2}}} h^{h}\left(x, t^{n}+\Delta t\right) d x .
$$

We immediately deduce that $h_{i}^{n+1} \geq 0$.

3.3. A class of relaxation model. The characterization of the relaxation model (3.1) was dictated by the definition of the VFRoe scheme. Following the same strategy, many other relaxation models can be considered. For instance, the reader is referred to $[7,3,12]$ where Suliciu relaxation schemes are derived. These schemes are accurate and stable but turn out to be, sometime, sophisticated in their implementation. 
The aim of the present paper is to derive schemes which must be easy to implement, like the VFRoe method. Following the same idea, other relaxation methods, involving easy linearization, can be proposed. For instance, let us consider the following relaxation model:

$$
\left\{\begin{array}{l}
\partial_{t} h+\partial_{x}\left(\frac{\Sigma^{2}}{g} U\right)=0, \quad t>0, x \in \mathbb{R}, \\
\partial_{t}(h u)+\partial_{x}\left(\frac{\Sigma^{2}}{2 g}\left(2 U^{2}+\Sigma^{2}\right)\right)=0, \\
\partial_{t} \Sigma+\frac{\lambda^{+}-\lambda^{-}}{\alpha+\beta} \partial_{x} U+\frac{\alpha \lambda^{-}+\beta \lambda^{+}}{\alpha+\beta} \partial_{x} \Sigma=\mu(c-\Sigma), \\
\partial_{t} U+\frac{\alpha \lambda^{+}+\beta \lambda^{-}}{\alpha+\beta} \partial_{x} U-\frac{\alpha \beta}{\alpha+\beta}\left(\lambda^{+}-\lambda^{-}\right) \partial_{x} \Sigma=\mu(u-U),
\end{array}\right.
$$

where $\alpha$ and $\beta$ are positive parameters to be fixed. This model is nothing but an extension of (3.1). Indeed, (3.27) coincides with (3.1) as soon as $\alpha=\beta=2$. Considering physical applications, a suitable choice of the parameters $\alpha$ and $\beta$ should give more accurate simulations.

We skip the algebra analysis of this system which turns out to be easy (see also the above section). After straightforward computations, the resulting scheme reads in the form (3.24) where the numerical flux function is given by (3.26). Only the definition of $\left(u^{*}, c^{*}\right)$ has changed and now are given by:

$$
\begin{aligned}
& c^{*}= \begin{cases}c_{L} & \text { if } \lambda^{-}>0, \\
\frac{\alpha c_{L}+\beta c_{R}}{\alpha+\beta}-\frac{1}{\alpha+\beta}\left(u_{R}-u_{L}\right) & \text { if } \lambda^{-}<0<\lambda^{+}, \\
c_{R} & \text { if } \lambda^{+}<0,\end{cases} \\
& u^{*}= \begin{cases}u_{L} & \text { if } \lambda^{-}>0, \\
\frac{\alpha u_{L}+\beta u_{R}}{\alpha+\beta}-\frac{\alpha \beta}{\alpha+\beta}\left(c_{R}-c_{L}\right) & \text { if } \lambda^{-}<0<\lambda^{+}, \\
u_{R} & \text { if } \lambda^{+}<0 .\end{cases}
\end{aligned}
$$

Once again, we can establish that the obtained updated depth $h_{i}^{n+1}$ remains nonnegative as soon as the relaxation wave speeds $\lambda^{-}$and $\lambda^{+}$are judiciously chosen.

4. High order extension. We turn considering extension of the above firstorder schemes to increase the order of accuracy. To access such an issue, we adopt the celebrate MUSCL method introduced by van Leer [29]. This technique is based on a linear (or higher order) reconstruction instead of piecewise constant approximation. This reconstruction allows to evaluate states $\mathbf{w}_{i+1, l}$ and $\mathbf{w}_{i, r}$ at each side of the interface located at $x_{i+\frac{1}{2}}$. The MUSCL scheme thus reads as follows:

$$
\mathbf{w}_{i}^{n+1}=\mathbf{w}_{i}^{n}-\frac{\Delta t}{\Delta x}\left(\mathbf{f}_{i+\frac{1}{2}}-\mathbf{f}_{i-\frac{1}{2}}\right)
$$

but, at this time, the numerical flux function involves the high order evaluated states at interfaces:

$$
\mathbf{f}_{i+\frac{1}{2}}=\mathbf{f}\left(\mathbf{w}_{i, r}, \mathbf{w}_{i+1, l}\right) .
$$

In order to enforce the required robustness of this modified scheme, several approaches can be considered. The most usual (see $[1,7,30]$ ) implies conservative reconstruction 
in the following sense:

$$
\frac{1}{2}\left(\mathbf{w}_{i, l}+\mathbf{w}_{i, r}\right)=\mathbf{w}_{i}^{n}
$$

As soon as the reconstructed states $\mathbf{w}_{i, l}$ and $\mathbf{w}_{i, r}$ stay in $\Omega$, involving a relevant half $\mathrm{CFL}$ restriction [7,30], the updated states preserve the non-negativity of the depth: $h_{i}^{n+1} \geq 0$. The assumption (4.3) appears as natural within the framework of second-order MUSCL scheme, where linear reconstruction are proposed. Now, when high-order reconstruction are suggested, the assumption (4.3) is too restrictive and cannot be assumed. After an idea introduced by Perthame-Shu [37] (see also some recent works $[4,5,6]$ ), relevant interface states can be evaluated in order to preserve the expected depth non-negativity when (4.3) is violated.

We assume that the reconstructed states $\mathbf{w}_{i, l}$ and $\mathbf{w}_{i, r}$ belong to $\Omega$ and satisfy the required order of accuracy. Following [4], we introduce an intermediate state, denoted $\mathbf{w}_{i}^{*}$, defined by

$$
\frac{1}{3}\left(\mathbf{w}_{i, l}+\mathbf{w}_{i}^{*}+\mathbf{w}_{i, r}\right)=\mathbf{w}_{i}^{n} .
$$

The reconstruction, or equivalently the characterization of $\mathbf{w}_{i, r}$ and $\mathbf{w}_{i, l}$, for all $i \in \mathbb{Z}$, must be done to enforce $\mathbf{w}_{i, l} \in \Omega, \mathbf{w}_{i, r} \in \Omega$ but also $\mathbf{w}_{i}^{*} \in \Omega$. Involving a relevant CFL condition, formally divided by three, the MUSCL scheme (4.1)-(4.2) preserves the nonnegativity of $h_{i}^{n+1}$. In other words, this procedure enforces the expected robustness property independently of the reconstruction process. The robustness result, stated in [4], can be applied in the present work. Thus we have:

THEOREM 4.1. Assume that the reconstruction is such that $\mathbf{w}_{i, l}, \mathbf{w}_{i}^{*}$ and $\mathbf{w}_{i, r}$ belong to $\Omega$ for all $i \in \mathbb{Z}$. Assume the $C F L$ like condition:

$$
\frac{\Delta t}{\Delta x} \max \left(\left|\lambda^{ \pm}\left(\mathbf{w}_{i, r}, \mathbf{w}_{i+1, l}\right)\right|,\left|\lambda^{ \pm}\left(\mathbf{w}_{i, l}, \mathbf{w}_{i}^{*}\right)\right|,\left|\lambda^{ \pm}\left(\mathbf{w}_{i}^{*}, \mathbf{w}_{i, r}\right)\right|\right) \leq \frac{1}{6},
$$

where $\lambda^{ \pm}\left(\mathbf{w}_{L}, \mathbf{w}_{R}\right):=\bar{u}\left(\mathbf{w}_{L}, \mathbf{w}_{R}\right) \pm \bar{c}\left(\mathbf{w}_{L}, \mathbf{w}_{R}\right)$ define the egeinvalues evaluated according to the depth non-negativity Lemma 3.2. Then the updated states $\left(\mathbf{w}_{i}^{n+1}\right)_{i \in \mathbb{Z}}$, defined by the MUSCL scheme (4.1)-(4.2), belong to $\Omega$.

Proof. Involving the robust first-order VFRoe scheme (2.5)-(2.9), the updated reconstructed states $\mathbf{w}_{i, l}, \mathbf{w}_{i, r}$ and $\mathbf{w}_{i}^{*}$ can be written as follows:

$$
\begin{aligned}
& \mathbf{w}_{i, l}^{n+1}=\mathbf{w}_{i, l}-\frac{\Delta t}{\Delta x / 3}\left(\mathbf{f}\left(\mathbf{w}_{i, l}, \mathbf{w}_{i}^{*}\right)-\mathbf{f}\left(\mathbf{w}_{i-1, r}, \mathbf{w}_{i, l}\right)\right), \\
& \mathbf{w}_{i}^{*, n+1}=\mathbf{w}_{i}^{*}-\frac{\Delta t}{\Delta x / 3}\left(\mathbf{f}\left(\mathbf{w}_{i}^{*}, \mathbf{w}_{i, r}\right)-\mathbf{f}\left(\mathbf{w}_{i, l}, \mathbf{w}_{i}^{*}\right)\right), \\
& \mathbf{w}_{i, r}^{n+1}=\mathbf{w}_{i, r}-\frac{\Delta t}{\Delta x / 3}\left(\mathbf{f}\left(\mathbf{w}_{i, r}, \mathbf{w}_{i+1, l}\right)-\mathbf{f}\left(\mathbf{w}_{i}^{*}, \mathbf{w}_{i, r}\right)\right) .
\end{aligned}
$$

Under the CFL restriction (4.4), we immediately deduce from Theorem 3.3 that the three updated states belong to $\Omega$. We have just to note that the state $\mathbf{w}_{i}^{n+1}$, defined by (4.1)-(4.2), writes as follows:

$$
\mathbf{w}_{i}^{n+1}=\frac{1}{3}\left(\mathbf{w}_{i, l}^{n+1}+\mathbf{w}_{i}^{*, n+1}+\mathbf{w}_{i, r}^{n+1}\right),
$$

and the proof is completed. 
¿From a practical point of view, the reconstructed states at the interface read:

$$
\mathbf{w}_{i, l}=\mathbf{w}_{i}^{n}+\Delta_{i}^{-} \quad \text { and } \quad \mathbf{w}_{i, r}=\mathbf{w}_{i}^{n}+\Delta_{i}^{+},
$$

where $\Delta_{i}^{ \pm}$are the reconstruction increments. When considering conservative reconstructions, we have $\Delta_{i}^{-}+\Delta_{i}^{+}=0$. Now, as soon as $\Delta_{i}^{-}+\Delta_{i}^{+} \neq 0$, we have to introduce the intermediate sate $\mathbf{w}_{i}^{*}$, defined by:

$$
\mathbf{w}_{i}^{*}=\mathbf{w}_{i}^{n}-\left(\Delta_{i}^{-}+\Delta_{i}^{+}\right) .
$$

Concerning the depth evaluation, the increments are modified to enforce the following restrictions:

$$
h_{i}^{n}+(\Delta h)_{i}^{-} \geq 0, \quad h_{i}^{n}+(\Delta h)_{i}^{+} \geq 0, \quad h_{i}^{n}-\left((\Delta h)_{i}^{-}+(\Delta h)_{i}^{+}\right) \geq 0 .
$$

To illustrate this new restriction, we propose to introduce the following modified limitation:

$$
(\Delta h)_{i}^{-, l i m}=\theta \max \left((\Delta h)_{i}^{-},-h_{i}^{n}\right), \quad(\Delta h)_{i}^{+, l i m}=\theta \max \left((\Delta h)_{i}^{+},-h_{i}^{n}\right),
$$

where we have set

$$
\theta=\left\{\begin{array}{l}
1, \quad \text { if } \max \left((\Delta h)_{i}^{-},-h_{i}^{n}\right)+\max \left((\Delta h)_{i}^{+},-h_{i}^{n}\right) \leq 0, \\
\min \left(1, \frac{h_{i}^{n}}{\max \left((\Delta h)_{i}^{-},-h_{i}^{n}\right)+\max \left((\Delta h)_{i}^{+},-h_{i}^{n}\right)}\right), \quad \text { otherwise. }
\end{array}\right.
$$

Once this step is performed, the high order reconstructed variables are defined by (4.5) but for the limited increments $\Delta^{-, \lim }$ and $\Delta^{+, \lim }$. This additional limitation process is more sophisticated than standard reconstruction but it satisfies the condition (4.6). Of course other slope limitations can be suggested.

To conclude this brief robust MUSCL scheme presentation, let us note that the additional limitation preserves the order of accuracy of the interface states as soon as the exact solution at time $t^{n}, \mathbf{w}\left(x, t^{n}\right)$, is smooth enough.

To illustrate our purpose, a fourth-order MUSCL reconstruction is proposed in the present work and used in the numerical investigations of Section 6. Following [43], we set, for an arbitrary variable $q$ :

$$
(\Delta q)_{i}^{-}=-\frac{1}{6}\left(2 \Delta^{*} \bar{q}_{i-\frac{1}{2}}+\Delta^{*} \tilde{q}_{i+\frac{1}{2}}\right), \quad(\Delta q)_{i}^{+}=\frac{1}{6}\left(\Delta^{*} \bar{q}_{i-\frac{1}{2}}+2 \Delta^{*} \tilde{q}_{i+\frac{1}{2}}\right),
$$

where

$$
\begin{aligned}
& \Delta^{*} \bar{q}_{i-\frac{1}{2}}=\operatorname{minmod}\left(\Delta^{*} q_{i-\frac{1}{2}}, b \Delta^{*} q_{i+\frac{1}{2}}\right), \\
& \Delta^{*} \tilde{q}_{i+\frac{1}{2}}=\operatorname{minmod}\left(\Delta^{*} q_{i+\frac{1}{2}}, b \Delta^{*} q_{i-\frac{1}{2}}\right)
\end{aligned}
$$

and

$$
\begin{aligned}
& \Delta^{*} q_{i+\frac{1}{2}}=\Delta q_{i+\frac{1}{2}}-\frac{1}{6} \Delta^{3} \bar{q}_{i+\frac{1}{2}}, \\
& \Delta^{3} \bar{q}_{i+\frac{1}{2}}=\Delta \bar{q}_{i-\frac{1}{2}}-2 \Delta \bar{q}_{i+\frac{1}{2}}+\Delta \bar{q}_{i+\frac{3}{2}}, \\
& \Delta \bar{q}_{i-\frac{1}{2}}=\operatorname{minmod}\left(\Delta q_{i-\frac{1}{2}}, c \Delta q_{i+\frac{1}{2}}, c \Delta q_{i+\frac{3}{2}}\right), \\
& \Delta \bar{q}_{i+\frac{1}{2}}=\operatorname{minmod}\left(\Delta q_{i+\frac{1}{2}}, c \Delta q_{i+\frac{3}{2}}, c \Delta q_{i-\frac{1}{2}}\right), \\
& \Delta \bar{q}_{i+\frac{3}{2}}=\operatorname{minmod}\left(\Delta q_{i+\frac{3}{2}}, c \Delta q_{i-\frac{1}{2}}, c \Delta q_{i+\frac{1}{2}}\right),
\end{aligned}
$$


with

$$
\Delta q_{i+\frac{1}{2}}=q_{i+1}-q_{i}
$$

and

$$
1<b \leq 4, \quad c=2
$$

Concerning the numerical experiments proposed in the related section, the parameter $\mathrm{b}$ will be set to 4 . It is worth mentioning that this $4^{\text {th }}$-order reconstruction reduces to a classical $3^{r d}$-order scheme when replacing $\Delta^{*} q_{i+\frac{1}{2}}$ by $\Delta q_{i+\frac{1}{2}}$ in (4.9), as developed in [43]. Considering the shallow water system (1.1), one has to reconstuct conservative variables $h$ and $h u$ following (4.8), (4.9), (4.10), and to apply the modified limitation (4.7) only on $h$. Note that when considering well-balancing discretization of system (1.1), one has also to reconstruct and apply (4.7) on $H=h+d$ to preserve steady states at rest, as developed in the next section.

5. Well-balancing for source terms. In this section we focus on the shallow water system with a bed slope source term (1.1).

5.1. Hydrostatic reconstruction. We recall here how to obtain a high order well-balanced scheme satisfying the preservation of steady states "at rest", following the lines of the method proposed first in [1] and extended to higher order of accuracy in $[36]$.

The first step is to build a high-order reconstruction of the values at each side of the mesh interfaces. We find various ways to achieve such accuracy, including MUSCL reconstructions [43] (see also the above section) or ENO/WENO polynomial reconstructions $[42,36]$. Note that the main limitation of all these methods concerns the ability to deal with the occurrence of dry areas. In the following, we use the $4^{\text {th }}$ order MUSCL reconstruction (4.8), together with the modified limitation (4.7) to ensure the preservation of the water depth positivity within the reconstruction. Considering the cell $i$, we compute first reconstructions $\mathbf{w}_{i, r}$ and $\mathbf{w}_{i, l}$ respectively located at $x_{i+\frac{1}{2}-}$ and $x_{i-\frac{1}{2}+}$. Values of $H_{i, l}$ and $H_{i, r}$, where $H=h+d$, are also reconstructed, and we deduce reconstructions of the topography $d_{i, l}=H_{i, l}-h_{i, l}$ and $d_{i, r}=H_{i, r}-h_{i, r}$. It ensures that if $u_{i}=0$ and $H_{i}=H_{i+1}$ for all $i$, then $u_{i, l}=u_{i, r}=0$ and $H_{i, l}=H_{i, r}=H_{i}$ for all $i$. Note that even if the VFRoe solver introduced in Section 1 is defined with non-conservative variables, numerical investigations have shown that the choice of reconstructing conservative variables gives better results in practice.

In a second step, we perform the "hydrostatic reconstruction" of the values at each side of the mesh interfaces, taking into account the variations of the bottom and the balance obtained static flows. Interface topography values $d_{i+\frac{1}{2}}$ are defined as follows:

$$
d_{i+\frac{1}{2}}=\max \left(d_{i, r}, d_{i+1, l}\right) .
$$

Then, the reconstruction of the water height on each side of the considered interface is defined as follows:

$$
h_{i+\frac{1}{2}-}=\max \left(0, h_{i, r}+d_{i, r}-d_{i+\frac{1}{2}}\right), \quad h_{i+\frac{1}{2}+}=\max \left(0, h_{i+1, l}+d_{i+1, l}-d_{i+\frac{1}{2}}\right),
$$

and we deduce from it the complete reconstructed values on each side of the interface:

$$
\mathbf{w}_{i+\frac{1}{2}-}=\left(\begin{array}{c}
h_{i+\frac{1}{2}-} \\
h_{i+\frac{1}{2}-} u_{i, r}
\end{array}\right), \quad \mathbf{w}_{i+\frac{1}{2}+}=\left(\begin{array}{c}
h_{i+\frac{1}{2}+} \\
h_{i+\frac{1}{2}+} u_{i+1, l}
\end{array}\right) .
$$


Then, using these new reconstructed values to compute the interface solution within a VFRoe formalism and with the notations introduced in Section 2, the numerical flux $\mathbf{f}_{i+\frac{1}{2}, j}^{*}$ is defined as follows:

$$
\mathbf{f}_{i+\frac{1}{2}}^{*}=\mathbf{f}\left(\mathcal{W}\left(\mathbf{u}^{*}\left(0 ; \mathcal{U}\left(\mathbf{w}_{i+\frac{1}{2}-}\right), \mathcal{U}\left(\mathbf{w}_{i+\frac{1}{2}+}\right)\right)\right)\right.
$$

Motivated by balancing requirements for static flows, the source term $\mathbf{S}_{i}$ is discretized and distributed to the cell interfaces, using both high order and hydrostatic reconstructed values of the water height:

$$
\mathbf{S}_{i}=\mathbf{S}_{i+\frac{1}{2}-}+\mathbf{S}_{i-\frac{1}{2}+}=\left(\begin{array}{c}
0 \\
\frac{g}{2} h_{i+\frac{1}{2}-}^{2}-\frac{g}{2} h_{i, r}^{2}
\end{array}\right)+\left(\begin{array}{c}
0 \\
\frac{g}{2} h_{i, l}^{2}-\frac{g}{2} h_{i-\frac{1}{2}+}^{2}
\end{array}\right) .
$$

A centered source term $S_{c, i}$ is added to preserve consistency and well-balancing:

$$
\mathbf{S}_{c, i}=\left(\begin{array}{c}
0 \\
g \frac{h_{i, l}+h_{i, r}}{2}\left(d_{i, l}-d_{i, r}\right)
\end{array}\right)
$$

It leads to the following semi-discrete formulation:

$$
\frac{d}{d t} \mathbf{w}_{i}(t)+\frac{1}{\Delta x}\left(\mathbf{f}_{i+\frac{1}{2}}^{-}-\mathbf{f}_{i-\frac{1}{2}}^{+}\right)=\mathbf{S}_{c, i} .
$$

with left and right numerical fluxes through the mesh interfaces defined as follows:

$$
\begin{aligned}
\mathbf{f}_{i+\frac{1}{2}}^{-} & =\mathbf{f}_{i+\frac{1}{2}}^{*}+\mathbf{S}_{i+\frac{1}{2}-} \\
& =\mathbf{f}\left(\mathcal{W}\left(\mathbf{u}^{*}\left(0 ; \mathcal{U}\left(\mathbf{w}_{i+\frac{1}{2}-}\right), \mathcal{U}\left(\mathbf{w}_{i+\frac{1}{2}+}\right)\right)\right)+\left(\begin{array}{c}
0 \\
\frac{g}{2} h_{i, r}^{2}-\frac{g}{2} h_{i+\frac{1}{2}-}^{2}
\end{array}\right)\right. \\
\mathbf{f}_{i+\frac{1}{2}}^{+} & =\mathbf{f}_{i+\frac{1}{2}}^{*}+\mathbf{S}_{i+\frac{1}{2}+} \\
& =\mathbf{f}\left(\mathcal{W}\left(\mathbf{u}^{*}\left(0 ; \mathcal{U}\left(\mathbf{w}_{i+\frac{1}{2}-}\right), \mathcal{U}\left(\mathbf{w}_{i+\frac{1}{2}+}\right)\right)\right)+\left(\begin{array}{c}
\frac{g}{2} h_{i+1, l}^{2}-\frac{g}{2} h_{i+\frac{1}{2}+}^{2}
\end{array}\right) .\right.
\end{aligned}
$$

5.2. Well-balancing for steady states. We briefly recall the proof for the well-balanced property. Assuming that $H=h+d$ is constant at time $t$ and that $u=0$, we have $H_{i, r}=H_{i, l}$ and

$$
h_{i+\frac{1}{2}-}=\max \left(0, H_{i, r}-d_{i+\frac{1}{2}}\right)=\max \left(0, H_{i, l}-d_{i+\frac{1}{2}}\right)=h_{i+\frac{1}{2}+},
$$

and by construction:

$$
\mathbf{w}_{i+\frac{1}{2}-}=\left(\begin{array}{c}
h_{i+\frac{1}{2}-} \\
0
\end{array}\right)=\left(\begin{array}{c}
h_{i+\frac{1}{2}+} \\
0
\end{array}\right)=\mathbf{w}_{i+\frac{1}{2}+} .
$$

Considering (5.4) and (5.8), we obtain:

$$
\mathbf{f}_{i+\frac{1}{2}}^{-}=\left(\begin{array}{c}
0 \\
\frac{g}{2} h_{i, r}^{2}
\end{array}\right) \quad \text { and } \quad \mathbf{f}_{i-\frac{1}{2}}^{+}=\left(\begin{array}{c}
0 \\
\frac{g}{2} h_{i, l}^{2}
\end{array}\right)
$$


and the semi-discrete formulation (5.7) leads to the following identity:

$$
\Delta x \frac{d}{d t}\left(\begin{array}{c}
h_{i}(t) \\
\left(h_{i} u_{i}\right)(t)
\end{array}\right)+\left(\begin{array}{c}
0 \\
\frac{g}{2} h_{i, r}^{2}
\end{array}\right)-\left(\begin{array}{c}
0 \\
\frac{g}{2} h_{i, l}^{2}
\end{array}\right)=\left(\begin{array}{c}
0 \\
g \frac{h_{i, l}+h_{i, r}}{2}\left(d_{i, l}-d_{i, r}\right)
\end{array}\right) .
$$

Therefore, we have:

$\Delta x \frac{d}{d t}\left(h_{i} u_{i}\right)(t)=g \frac{h_{i, l}+h_{i, r}}{2}\left(h_{i, l}-h_{i, r}\right)+g \frac{h_{i, l}+h_{i, r}}{2}\left(\left(H_{i, l}-h_{i, l}\right)-\left(H_{i, r}-h_{i, r}\right)\right)$,

and

$$
\Delta x \frac{d}{d t}\left(h_{i} u_{i}\right)(t)=g \frac{h_{i, l}+h_{i, r}}{2}\left(H_{i, r}-H_{i, l}\right)=0 .
$$

The expected well-balanced property is thus satisfied.

6. Numerical results. In the following test cases, we use a fourth order accurate scheme, based on the stabilized VFRoe scheme introduced in Section 2 . The $4^{\text {th }}$ order MUSCL reconstruction (4.8) together with the new modified limitation procedure (4.7) are considered. The hydrostatic reconstruction recalled in Section 5 is used to account for topography variations in a well-balanced way. The semi-discrete discretization (5.7) is replaced by a $3^{\text {rd }}$ order TVD Runge-Kutta time discretization [38]. To assess the improvements of accuracy obtained with this high order reconstruction, we begin in the first two cases to perform a comparison with the present scheme and classical first and second order VFRoe schemes. In all the following cases, we only show numerical results obtained with the present $4^{\text {th }}$-order scheme, in situations involving steady states and occurrences of dry states. The CFL are respectively set to $0.5,0.25$ and 0.15 for first, second and fourth order reconstructions, in agreement with the stability analysis performed in the above sections. Let us emphasize that, according to the work of Masella et al. [35] (see also [20]), the present scheme is modified in order to handle the sonic rarefaction wave invoking the standard entropy correction.

6.1. Sinusoidal wave propagation in shallow water. This first test describes a sinusoidal wave propagating in a channel with a horizontal bottom, taken from [22]. The channel is $A=1000 \mathrm{~m}$ long and the still water depth is $H_{0}=10 \mathrm{~m}$. The period and amplitude of the sinusoidal wave are $20.193 \mathrm{~s}$ and $0.04 \mathrm{~m}$, respectively. In this case, the ratio $A / H_{0} \approx 0.016$ is close to the limit of shallow water conditions. Note that this test differs from the original one introduced in [22], since we used an absorbing boundary [41] at the right boundary, in order to let the incoming wave exit the computational domain without reflexions. The channel is divided into 250 cells and the propagation is performed respectively with schemes of first, second and fourth order of accuracy, to clearly exhibit the numerical diffusion induced by lower order methods. We obtain a steady wave solution with a wavelength of $200 \mathrm{~m}$, which is in agreement with the expected wavelength. As can be seen in Figure 6.1 , the $4^{\text {th }}$ order stabilized MUSCL reconstruction largely reduces the numerical diffusion observed with $1^{\text {st }}$ and $2^{\text {nd }}$ order schemes, even with a small number of cells.

6.2. Subcritical flow over a bump. In this test, the bottom is defined as follows:

$$
d(x)= \begin{cases}0.2-0.05(x-10)^{2}, & \text { if } 8 m<x<12 m \\ 0, & \text { else }\end{cases}
$$




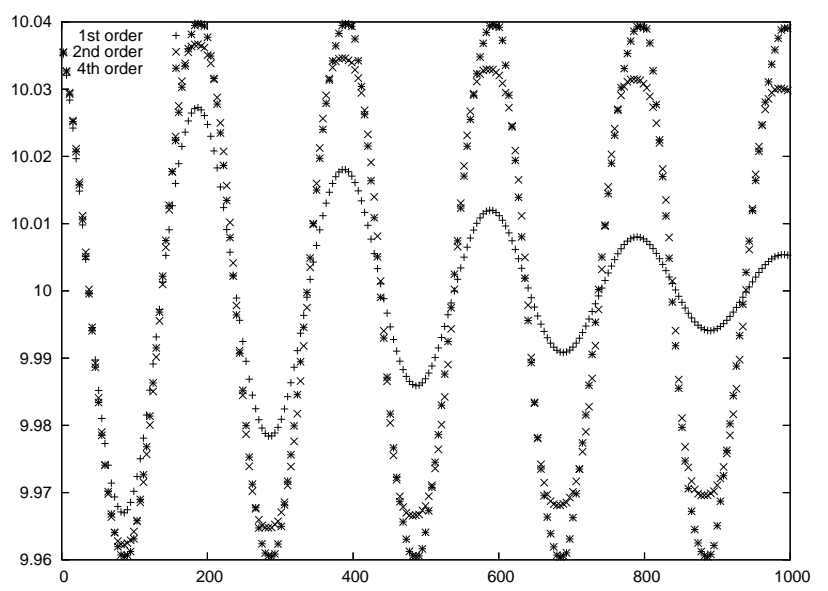

FIG. 6.1. Sinusoidal wave propagation in shallow water. Free surface evolution: comparison between $1^{\text {st }}, 2^{\text {nd }}$ and $4^{\text {th }}$ order schemes for 250 cells.

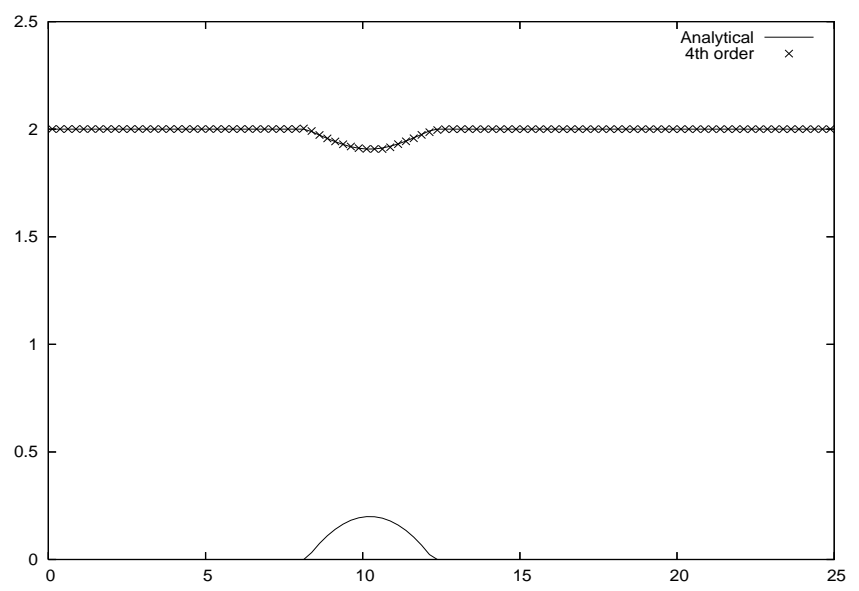

FIG. 6.2. Subcritical flow over a bump: water height at steady state.

and we consider a channel of $25 \mathrm{~m}$ long. The boundary conditions are $h_{\text {out }}=2 \mathrm{~m}$ and $Q_{i n}=4.42 \mathrm{~m}^{2} / \mathrm{s}$, corresponding to a sub-critical flow. We obtain a stationary solution, shown on Figure 6.2. We observe on this Figure the numerical results obtained with the $4^{\text {th }}$ order scheme at time $T=200 \mathrm{~s}$ with 100 cells, superposed with the analytical solution. Since the results obtained with $1^{\text {st }}, 2^{\text {nd }}$ and $4^{\text {th }}$ order schemes are similar once the convergence is achieved, we also show on Figure 6.3 a comparison between $L^{2}$-error time-series for each order of accuracy. We can clearly observe the convergence toward the steady state at rest in the three cases. For scaling reasons, the transient part of the computation is partially truncated and we only show on Figure 6.3 time series of $L^{2}$-error for values of time from $t=50 \mathrm{~s}$ and $t=200 \mathrm{~s}$. 


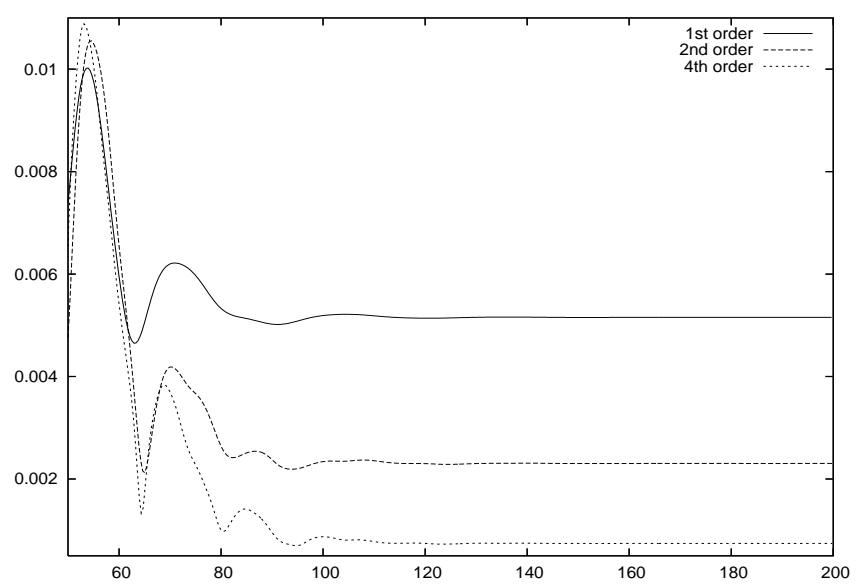

FIG. 6.3. Subcritical flow over a bump: $L^{2}$-error time series, comparison between $1^{\text {st }}, 2^{\text {nd }}$ and $4^{\text {th }}$ order.

Lastly, we show the $L^{2}$-errors table, computed for the water height, for various numbers of cells, at $t=200 \mathrm{~s}$ :

\begin{tabular}{|c|c|c|c|}
\hline cells & $1^{\text {st }}$ order scheme & $2^{\text {nd }}$ order scheme & $4^{\text {th }}$ order scheme \\
\hline 50 & $5.155 \mathrm{E}-3$ & $1.700 \mathrm{E}-3$ & $7.422 \mathrm{E}-4$ \\
\hline 100 & $2.704 \mathrm{E}-3$ & $6.062 \mathrm{E}-4$ & $1.961 \mathrm{E}-4$ \\
\hline 200 & $1.383 \mathrm{E}-3$ & $1.759 \mathrm{E}-4$ & $6.155 \mathrm{E}-5$ \\
\hline 400 & $6.993 \mathrm{E}-4$ & $5.730 \mathrm{E}-5$ & $2.103 \mathrm{E}-5$ \\
\hline 800 & $3.516 \mathrm{E}-4$ & $1.950 \mathrm{E}-5$ & $7.242 \mathrm{E}-6$ \\
\hline 1600 & $1.763 \mathrm{E}-4$ & $6.787 \mathrm{E}-6$ & $1.562 \mathrm{E}-6$ \\
\hline
\end{tabular}

We can observe some improvements obtained with the $4^{\text {th }}$ order reconstruction proposed. However, it is worth mentionning that we have only performed here a $2^{\text {nd }}$ order discretization of the source term, which lowered the overall accuracy. A better accuracy can of course be obtained using a stabilized high order reconstruction together with a high order quadrature discretization rule for the source term, like the one introduced in the recent work [36].

We conclude the analysis of the present numerical experiment with a comparison of the efficiency of both $2^{\text {nd }}$ and $4^{\text {th }}$ order methods. To consider a similar $L^{2}$-error at time $t=200 \mathrm{~s}$, we adopt the $2^{\text {nd }}$ order scheme with 1600 cells while the $4^{\text {th }}$ order method is used with 800 cells. Invoking the CPU time for these two tests, we obtain that the $4^{\text {th }}$ scheme is twice more quickly than the $2^{\text {nd }}$ order scheme.

6.3. Flow at rest with dry state. The initial condition of this test is a flow at rest, involving a dry area. We impose $h+d=\max (d, 0.15) \mathrm{m}$ and $Q=0 \mathrm{~m}^{2} / \mathrm{s}$ over the whole channel. The bottom variations are defined with (6.1). Numerical results, obtained with 100 cells at time $T=200 \mathrm{~s}$ are plotted on Figure 6.4 with and without the modified limitation (4.7) (respectively in solid and dashed lines). We can clearly show that the stabilized $4^{\text {th }}$ order scheme proposed here is well-balanced and can handle dry areas whereas large spurious oscillations are rapidly generated when stabilization (4.7) is not applied. 


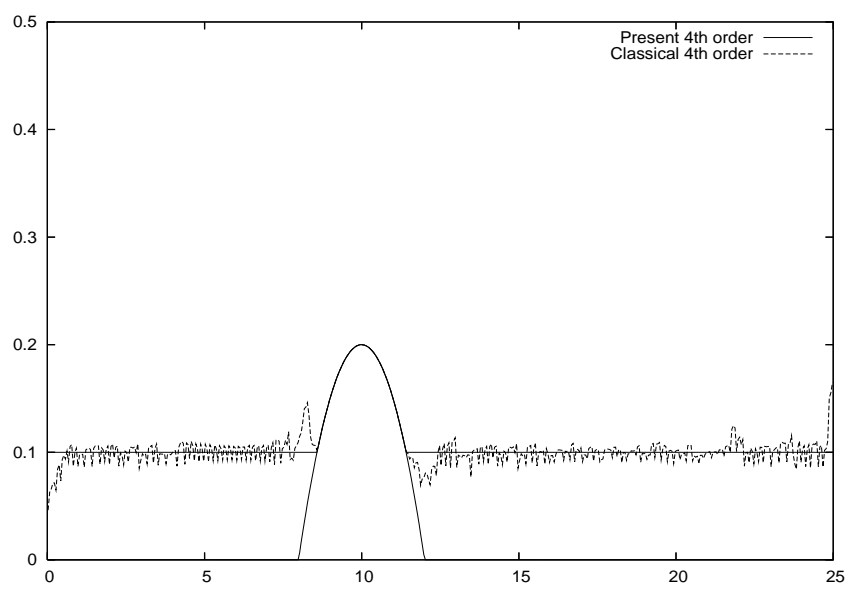

FIG. 6.4. Flow at rest with a dry state: water height. Comparison between stabilized $4^{\text {th }}$-order and classical $4^{\text {th }}$ order.

6.4. Drain on a non-flat bottom. The length of the channel and the topography of this test are the same as in the two previous cases. The initial condition here is a flow at rest, with a water heigth given by $h+d=0.5 \mathrm{~m}$ such that the bump is entirely submerged and the initial discharge is set to $Q=0 \mathrm{~m}^{2} / \mathrm{s}$ over the whole domain. For this more complex test, the whole domain is discretized into 500 cells. The left boundary is a solid-wall boundary (defined within a classical "mirror-state" procedure) and an outlet condition on a dry bed [13] at the right boundary. With such a configuration, the water progressively exits the computational domain, asymptotically converging towards a steady state composed of a state at rest on the left part of the bump with $h+d=0.2 \mathrm{~m}$ and $Q=0 \mathrm{~m}^{2} / \mathrm{s}$ and a dry state on the right side of the bump. Results obtained with the $4^{\text {th }}$ order stabilized scheme are shown on Figure 6.5 for the water height and on Figure 6.6 for the discharge. These results are qualitatively similar to those obtained with the second order scheme introduced in [13]. We emphasize that the use of our stabilized $4^{t h}$-order reconstruction (4.7) allows accurate results even during the draining part of the simulation and that we accurately converge toward the expected steady state. We can observe the generation of tiny oscillations near the discontinuities.

6.5. Vacuum occurrence by a double rarefaction wave over a step. In this last test, inroduced in [13], we do not study the convergence toward a steady state but we rather focus on the ability of the proposed stabilized $4^{\text {th }}$ order wellbalanced scheme to deal with dry areas over a discontinuous bottom. The topography is different from previous tests and is defined as follows:

$$
d(x)= \begin{cases}1, & \text { if } 8.33 m<x<12.5 m \\ 0, & \text { else }\end{cases}
$$

whereas the channel length is still $25 \mathrm{~m}$, divided into 500 cells. The initial condition is set to $h+d=10 \mathrm{~m}$ and the initial discharge is defined as follows:

$$
Q(x)= \begin{cases}-350 \mathrm{~m}^{2} / \mathrm{s}, & \text { if } x<50 / 3 \mathrm{~m} \\ 350 \mathrm{~m}^{2} / \mathrm{s}, & \text { otherwise. }\end{cases}
$$




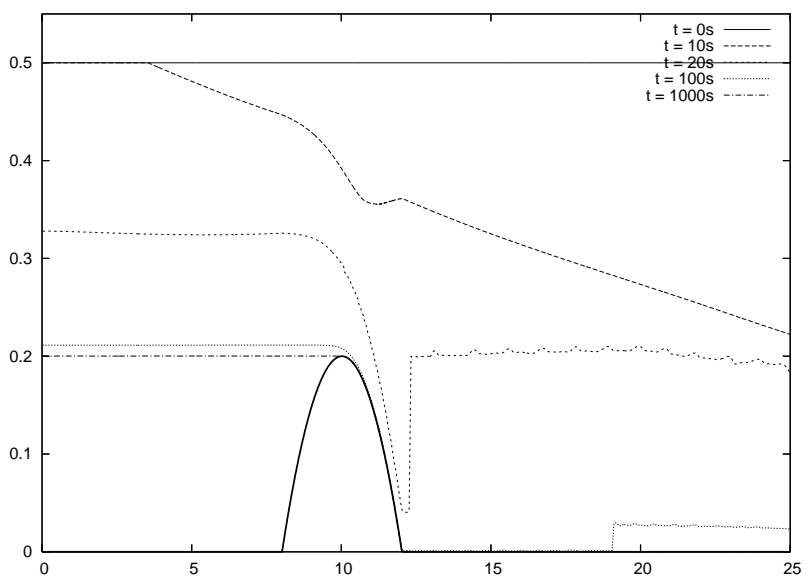

FIG. 6.5. Drain on a non-flat bottom: water height.

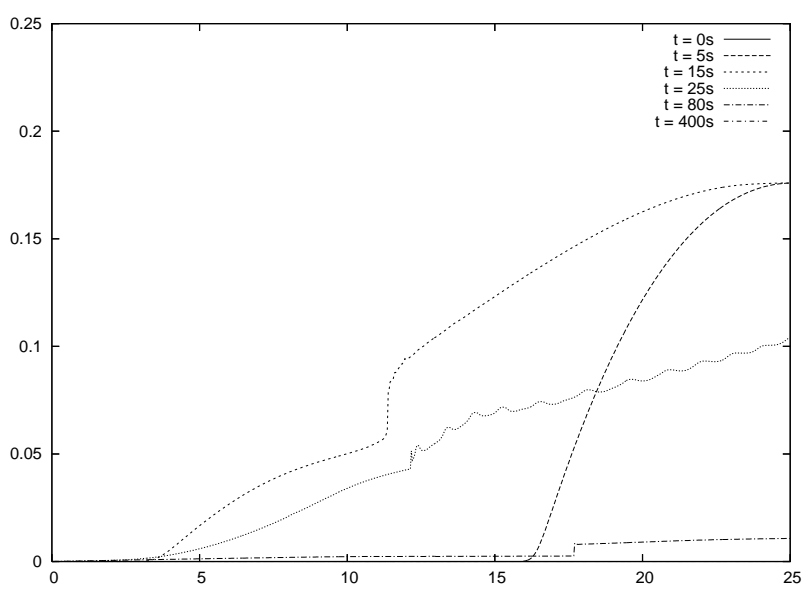

FIG. 6.6. Drain on a non-flat bottom: discharge.

Results at several times are shown on Figure 6.7 and Figure 6.8 respectively for the free surface and the discharge, at time $0,0.10,0.30,0.40$ and $0.60 \mathrm{~s}$. For the sake of clarity, we do not show the results obtained with $1^{\text {st }}$ and $2^{\text {nd }}$-order schemes but we stress out that the results obtained here with our stabilized $4^{\text {th }}$-order scheme are qualitatively very similar. The main difference lies in the occurrence of tiny oscillations near discontinuities for the water height, as observable on Figure 6.7.

6.6. The Carrier and Greenspan transient solution. We propose here to study one of the test cases developped in [34] to highlight the improvement obtained with the stabilized $4^{\text {th }}$ order approach even in situations involving complex drying and flooding processes. The reader is refered to [34] or to the original paper [10] for a detailed description. Note that an analytical solution is provided in [10]. In this test, the initial water surface elevation is assumed to be depressed near the shoreline, defined as the frontier between the fluid domain and the dry area, and the fluid held motionless. Then, the fluid is released at $t=0$ and we mainly focus on the shoreline evolution, During the evolution, the shoreline rises above the mean sea level of value 


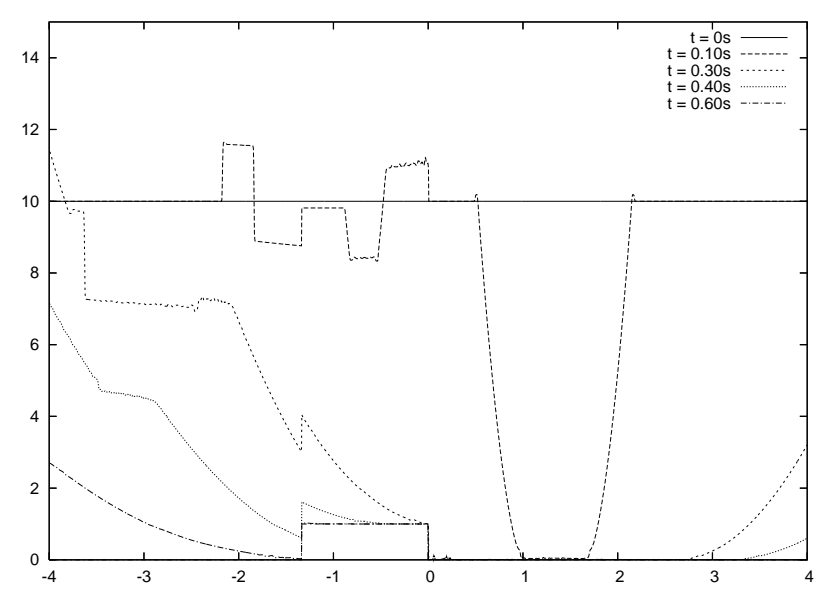

FIG. 6.7. Vacuum occurrence by a double rarefaction wave over a step : water height.

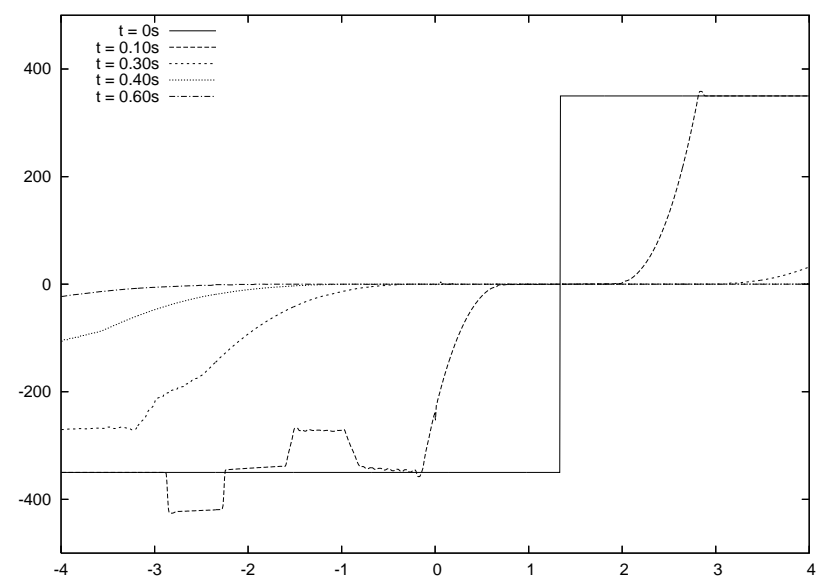

FIG. 6.8. Vacuum occurrence by a double rarefaction wave over a step : discharge.

$e$ and then the water surface elevation asymptotically settles back to it. This initial condition is the lower curve on Fig. 6.9. Let $l$ be the typical length scale of this specific problem and $\alpha$ the beach slope. Non-dimensional variables are defined as follows :

$$
x^{*}=x / l, \quad h^{*}=h /(\alpha l), \quad u^{*}=u / \sqrt{g \alpha l}, \quad t^{*}=t / \sqrt{l / \alpha g} .
$$

The bottom slope $\alpha$ is taken to be $1 / 50$, the results are presented here for $e=0.1$ and the initial surface profile is imposed in the dimensional case with the length scale $l=20 \mathrm{~m}$. Note that the CFL is set to 0.7 for the $1^{\text {st }}$ and $2^{\text {nd }}$ order schemes and to 0.3 for the stabilized $4^{\text {th }}$ order scheme. Fig. 6.9 shows comparisons between numerical results obtained with the stabilized $4^{\text {th }}$ order scheme and the analytical solution for the surface elevation, at various values of time. These surface elevation profiles have been scaled with the parameter $e$. It is worth mentionning that the results introduced in [34] for this case and obtained with a second order well-balanced scheme were already very good. Thus a table of comparison of $L^{2}$-errors obtained for various orders and several numbers of cells is provided at $t^{*}=10$ : 


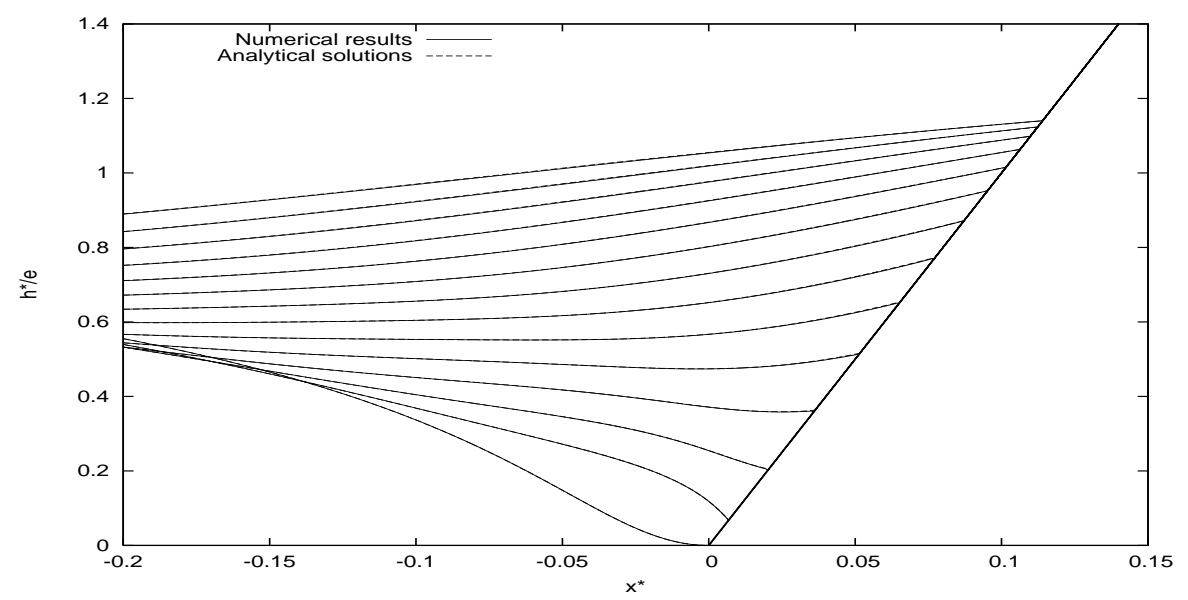

FIG. 6.9. The Carrier and Greenspan's transient solution. Comparison between numerical results (in solid lines) obtained with the $4^{\text {th }}$ and analytical solutions (in dotted lines) for the surface elevation. Profiles of water height $h^{*} / e$ are plotted versus $x^{*}$, for different values of time $t^{*}$, increasing from $t^{*}=0$ (bottom curves) to $t^{*}=1.4$ (top curves).

\begin{tabular}{|c|c|c|}
\hline cells & $2^{\text {nd }}$ order scheme & $4^{\text {th }}$ order scheme \\
\hline 250 & $3.095 \mathrm{E}-3$ & $5.912 \mathrm{E}-4$ \\
\hline 500 & $2.249 \mathrm{E}-3$ & $2.151 \mathrm{E}-4$ \\
\hline 1000 & $2.058 \mathrm{E}-3$ & $1.516 \mathrm{E}-4$ \\
\hline 2000 & $8.690 \mathrm{E}-4$ & $1.223 \mathrm{E}-5$ \\
\hline
\end{tabular}

From this table we observe that both schemes provide stability and good accuracy in the computation of the slow convergence toward the mean water level. The $L^{2}$ error computed for large values of time with the $2^{\text {nd }}$ order scheme seems to decrease very slowly with respect to the number of cells. This feature is slightly improved with the $4^{\text {th }}$ order scheme. It appears from this test that the stabilized $4^{\text {th }}$ order scheme provides more accuracy for large values of time.

6.7. Oblique 2D dam-break on a dry bed. We study in this last test the evolution of a mound of water over a flat bottom, which is suddenly released from an initial position, generating a propagating front such that the line of water propagates with an inclination of $45^{\circ}$ with respect to the boundaries of the computational domain. The base of this domain is a $[-0.5,0.5] \times[-0.5,0.5]$ square. The initial condition, shown at Figure 6.10 , is defined as follows :

$$
h(0, x, y)=\left\{\begin{array}{l}
h_{L} \text { for } x+y \leq 0 \\
0 \text { otherwise }
\end{array}\right.
$$

and the initial discharge is set to zero. The purpose of this last test is to shown the possibility of extension of the stabilized $4^{\text {th }}$-order scheme towards two-dimensional simulations. The results shown here have been obtained using 100 cells in each direction and the CFL is set to 0.2 . We only focus on the surface elevation computed on the central cross-section orthogonal to the propagating front (the $\mathrm{x}=\mathrm{y}$ plane) which is compared to the one-dimensional analytical solution, assuming that the effects induced by the boundaries can be neglected for this section and for a small time of 


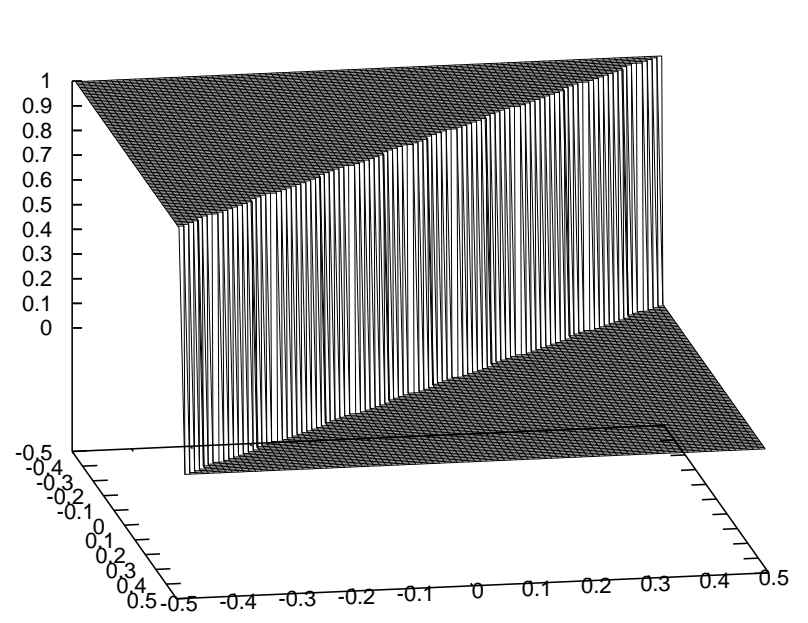

FIG. 6.10. Oblique 2D dam-break on a dry bed : initial surface elevation

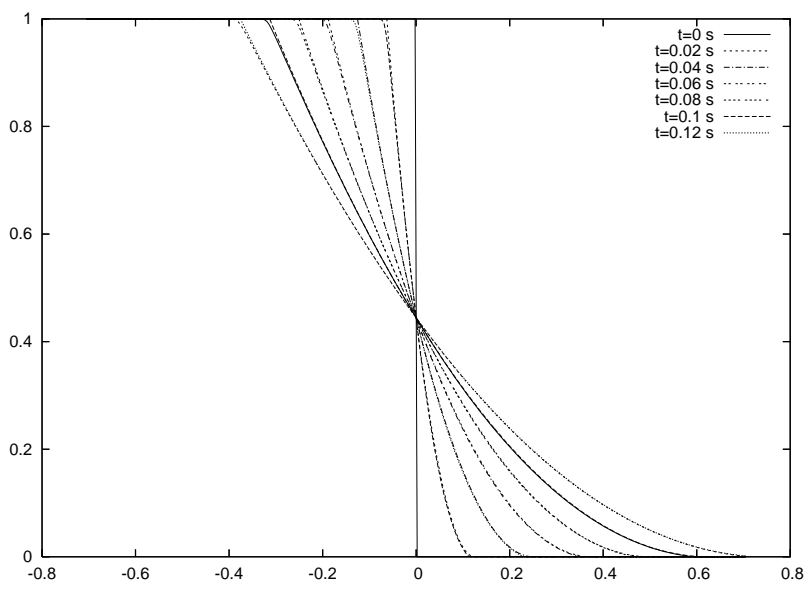

FIG. 6.11. Oblique 2D dam-break on a dry bed : time evolution of the surface elevation in the central cross section and comparison with the analytical $1 D$ solution

evolution. A comparison with the analytical solution in the direction of propagation is reported on Figure 6.11. The exact and approximated solutions are superposed. To conclude this $2 \mathrm{D}$ test, after $[5,16]$, let us note that unstructured mesh extensions can be performed.

7. Conclusion. In the present work, we have derived a numerical relaxation scheme to approximate the weak solutions of the shallow water equation. The analysis of the obtained approximate Riemann solver allows us to prove the required robustness results. Indeed, involving a suitable CFL like restriction, we establish the non-negativity of the approximated depth. In addition, the considered relaxation 
scheme is able to deal with dry areas and no blow-up is developed. In fact, the relaxation scheme under consideration exactly coincides with one variant of the well-known VFRoe scheme. As a consequence, we have established that the VFroe scheme does not produce negative value of the water depth. This statement is obtained when involving a relevant upwind technique. Next, we have proposed high-order extensions of this VFRoe scheme (second- and fourth-order) based on the van Leer slope reconstructions. Enforcing some suitable restriction in the gradient reconstruction procedure, we have proved that the considered high-order extensions, once again, preserve the non-negativity of the depth. The final well-balanced scheme is obtained when assuming the hydrostatic reconstruction to approximate the geometrical source terms. Several one-dimensional numerical experiments have shown the interest of the method. The extension towards two-dimensional simulations is introduced and currently under investigation for realistic problems.

\section{REFERENCES}

[1] E. Audusse, F. Bouchut, M. O. Bristeau, R. Klein, B. Perthame, A fast and stable well-balanced scheme with hydrostatic reconstruction for shallow water flows, SIAM J.Sci.Comp., 25, 2050-2065 (2004).

[2] M. Baudin, C. Berthon, F. Coquel, R. Masson, Q. H. Tran, A relaxation method for two-phase flow models with hydrodynamic closure law, Num. Math., 99, 411-440 (2005).

[3] C. Berthon, Numerical approximations of the 10-moment Gaussian closure, Math. Comput., 75, 1809-1831 (2006).

[4] C. Berthon, Stability of the MUSCL schemes for the Euler equations, Comm. Math. Sci., 3 , 133-158 (2005).

[5] C. Berthon, Robustness of MUSCL schemes for 2D unstructured meshes, J. Comput. Phys. 218, 495-509 (2006).

[6] C. Berthon, Why the MUSCL-Hancock scheme is $\mathrm{L}^{1}$-stable, Numer. Math., 104, $27-46$ (2006).

[7] F .Bouchut, Non-linear stability of finite volume methods for hyperbolic conservation laws and well-balanced schemes for sources, Frontiers in Mathematics, Birkhauser, 2004.

[8] T. Buffard, T. Gallouet, J.M. Herard, A naive Godunov scheme to solve shallow water equations, CR Acad. Sci. Paris, 326, 385-390 (1998).

[9] T. Buffard, T. Gallouet, J. M. Herard, A sequel to a rough Godunov scheme: application to real gases, Computers and Fluids, 29, 813-847 (2000).

[10] G.F. Carrier and H.P. Greenspan, Water waves of finite amplitude on a sloping beach, J. Fluid Mech., 4, 97-109 (1958).

[11] G.Q. Chen, C.D. Levermore, T.P. Liu, Hyperbolic Conservation Laws with Stiff Relaxation Terms and Entropy, Comm. Pure Appl. Math., 47, 787-830 (1995).

[12] F. Coquel and B. Perthame, Relaxation of Energy and Approximate Riemann Solvers for General Pressure Laws in Fluid Dynamics, SIAM J. Numer. Anal., 35, 2223-2249 (1998).

[13] T. Gallouet, J. M. Hérard, N.Seguin, Some approximate Godunov schemes to compute shallowwater equations with topography, Computers and Fluids, 32, 479-513 (2003).

[14] T. Gallouet, J. M. Hérard, N.Seguin, On the use of some symetrizing variables to deal with vacuum, Calcolo, 40, 163-194 (2003).

[15] T. Gallouët, T. Masella, A rough Godunov scheme, C. R., Math., Acad. Sci. Paris, 323, 77-83 (1996).

[16] E. Godlewski, P.A. Raviart, Numerical Approximation of Hyperbolic System of Conservation Laws, Applied Mathematical Sciences, 118, Springer, New-York, 1996.

[17] T.Gallouët, J.M. Hérard, N. Seguin, Some recent Finite Volume schemes to compute Euler equations using real gas EOS, Int J. Num. Meth. Fluids, 39, 1073-1138 (2002).

[18] J. M. Greenberg, A. Y. Leroux, A well-balanced scheme for the numerical processing of source terms in hyperbolic equations, SIAM J. Numer. Anal., 33, 1-16 (1996).

[19] J. M. Greenberg, A. Y. Leroux, R. Baraille, A. Noussair, Analysis and approximation of conservation laws with source terms, SIAM J. Numer. Anal., 34, 1980-2007 (1997).

[20] A. Harten, J. M. Hyman, P.D. Lax, On finite difference approximations and entropy conditions, Comm. Pure Applied Math., 29, 297-322 (1976).

[21] A. Harten, P.D. Lax, B. Van Leer, On upstream differencing and Godunov-type schemes for hyperbolic conservation laws, SIAM Review, 25, 35-61 (1983). 
[22] K. Hu, C.G. Mingham and D.M. Causon, Numerical simulation of wave overtopping of coastal structures using the non-linear shallow water equations, Coastal Engineering, 41, 433-465 (2000).

[23] S. Jin, A steady-state capturing method for hyperbolic systems with geometrical source terms, M2AN Math. Model. Numer. Anal., 35, 631-645 (2001).

[24] S. Jin, X. Wen, Two interface-type numerical methods for computing hyperbolic systems with geometrical source terms having concentrations, SIAM J. Sci. Comput., 26, 2079-2101 (2005).

[25] S. Jin, X. Wen, An efficient method for computing hyperbolic systems with geometrical source terms having concentrations, Special issue dedicated to the 70th birthday of Professor Zhong-Ci Shi. J. Comput. Math., 22, 230-249 (2004).

[26] S. Jin, Z. Xin, The Relaxation Scheme for Systems of Conservation Laws in Arbitrary Space Dimension, Comm. Pure Appl. Math., 45, 235-276 (1995).

[27] P. D. Lax, Hyperbolic systems of conservation laws and the mathematical theory of shock waves, Conference Board of the Mathematical Sciences Regional Conference Series in Applied Mathematics, 11, SIAM, Philadelphia (1973).

[28] P. D. Lax, Shock waves and entropy, Contributions to nonlinear functional analysis, E. H. Zarantonello, Ed., 603-634 (1971).

[29] B. van Leer, Towards the ultimate conservative difference scheme. V. A second-order sequel to Godunov's method, J. Comput. Phys., 32, 101-136 (1979).

[30] R. J. LeVeque, Finite volume methods for hyperbolic problems, Cambridge Texts in Applied Mathematics, Cambridge University Press, Cambridge (2002).

[31] R. J. LeVeque, M. Pelantiy, A class of approximate Riemann solvers and their relation to relaxation schemes, J. Comp. Phys., 172, 572-591 (2001)

[32] T.P. Liu, Hyperbolic conservation laws with relaxation, Comm. Math. Phys., 108, 153-175 (1987).

[33] F. Marche, Derivation of a new two-dimensional viscous shallow water model with varying topography, bottom friction and capillary effects, European Journal of Mechanic /B: Fluid, 26, 49-63 (2007).

[34] F. Marche, P. Bonneton, P. Fabrie, N. Seguin, Evaluation of well-balanced bore-capturing schemes for $2 D$ wetting and drying processes, Int. J. Numer. Meth. Fluids, 53, 867-894 (2007).

[35] J.M. Masella, I. Faille and T.Gallouet, On an approximate Godunov scheme, Int. J. Comp. Fluid. Dyn., 12, 133-149 (1999).

[36] S. Noelle, N. Pankratz, G. Puppo, J.R. Natvig, Well-balanced finite volume schemes of arbitrary order of accuracy for shallow water flows, J. Comp. Phys., 213, 474-499 (2006).

[37] B. Perthame, C.W. Shu, On positivity preserving finite volume schemes for Euler equations, Numer. Math., 73, 119-130 (1996).

[38] C.W. Shu, Total-variations-diminishing time discretization, SIAM J. Sci. Statist. Comp., 9, 1073-1084 (1988).

[39] I. Suliciu, On modelling phase transitions by means of rate-type constitutive equations, shock wave structure, Int. J. Engrg. Sci., 28, 829-841 (1990).

[40] I. Suliciu, Some stability-instability problems in phase transitions modelled by piecewise linear elastic or viscoelastic constitutive equations, Int. J. Engrg. Sci., 30, 483-494 (1992).

[41] G. Wei and J.T. Kirby, Time-dependant numerical code for extended Boussinesq equations, J. Water. Port. Coast. Ocean Engnr., 121, 251-261 (1995).

[42] Y. Xing, C.W. Shu, High order finite difference WENO schemes with the exact conservation property for the shallow water equations, J. Comp. Phys., 208, 206-227 (2005).

[43] S. Yamamoto, H. Daiguji, Higher order accurate upwind schemes for solving the compressible Euler and Navier-Stokes equations, Computers Fluids, 22, 259-270 (1993). 